\title{
The HR 4796A Debris System: Discovery of Extensive Exo-ring Dust Material
}

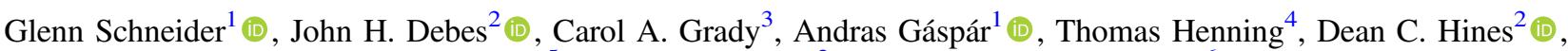 \\ Marc J. Kuchner ${ }^{5}$ (D), Marshall Perrin ${ }^{2}$ (D), and John P. Wisniewski ${ }^{6}$ (D) \\ ${ }^{1}$ Steward Observatory and the Department of Astronomy, The University of Arizona, 933 North Cherry Avenue, \\ Tucson, AZ 85721 USA; gschneider@as.arizona.edu \\ ${ }^{2}$ Space Telescope Science Institute, 3700 San Martin Drive, Baltimore, MD 21218 USA \\ ${ }^{3}$ Eureka Scientific, 2452 Delmer, Suite 100, Oakland, CA 96002 USA \\ ${ }^{4}$ Max-Planck-Institut für Astronomie, Königstuhl 17, D-69117, Heidelberg, Germany \\ ${ }^{5}$ NASA/Goddard Space Flight Center, Exoplanets \& Stellar Astrophysics Laboratory, Code 667, Greenbelt, MD 20771 USA \\ ${ }^{6}$ H. L. Dodge Department of Physics and Astronomy, University of Oklahoma, 440 West Brooks Street, Norman, OK 73019 USA \\ Received 2017 October 19; revised 2017 December 21; accepted 2017 December 21; published 2018 January 23
}

\begin{abstract}
The optically and IR-bright and starlight-scattering HR 4796A ringlike debris disk is one of the most- (and best-) studied exoplanetary debris systems. The presence of a yet-undetected planet has been inferred (or suggested) from the narrow width and inner/outer truncation radii of its $r=1$."05 (77 au) debris ring. We present new, highly sensitive Hubble Space Telescope (HST) visible-light images of the HR 4796A circumstellar debris system and its environment over a very wide range of stellocentric angles from 0 ." 32 (23 au) to $\approx 15^{\prime \prime}(1100 \mathrm{au})$. These very highcontrast images were obtained with the Space Telescope Imaging Spectrograph (STIS) using six-roll PSF template-subtracted coronagraphy suppressing the primary light of HR 4796A, with three image-plane occulters, and simultaneously subtracting the background light from its close angular proximity M2.5V companion. The resulting images unambiguously reveal the debris ring embedded within a much larger, morphologically complex, and biaxially asymmetric exo-ring scattering structure. These images at visible wavelengths are sensitive to and map the spatial distribution, brightness, and radial surface density of micron-size particles over 5 dex in surface brightness. These particles in the exo-ring environment may be unbound from the system and interacting with the local ISM. Herein, we present a new morphological and photometric view of the larger-than-prior-seen HR 4796A exoplanetary debris system with sensitivity to small particles at stellocentric distances an order of magnitude greater than has previously been observed.
\end{abstract}

Key words: ISM: general - methods: observational - stars: individual: HR 4796A

\section{Introduction}

Spatially resolved images of dusty debris in the circumstellar environments of nearby stars at visible to near-IR wavelengths reveal the distribution of small (micron-size) particles that may be sculpted by and co-orbiting with unimaged planets (e.g., Schneider et al. 2014, hereafter Sch14). Observable ringlike structures in such circumstellar debris disks seem to be a common feature (e.g., Bonnefoy et al. 2017; Feldt et al. 2017). The locations and surface brightness (SB) distributions of such rings may inform upon the architectures of these exoplanetary debris systems and offer constraints on planet masses and orbits even when the planets themselves remain undetected (e.g., Chiang et al. 2009; Rodigas et al. 2014; Thilliez \& Maddison 2016). More complex structures, e.g., arcs and spirals, seen in protoplanetary/transition disks around Herbig Ae stars are also observed (e.g., HD 141569; Konishi et al. 2016; Perrot et al. 2016), with remnant gas detected in some cases even at more advanced ages (e.g., Moór et al. 2017). The detection of diffuse starlight-scattering materials in large exoring debris halos that may be ejected or escaping these systems at large stellocentric distances due to forces posited both intrinsic and extrinsic has been technically challenging (e.g., Schneider et al. 2016, hereafter Sch16). Such images have been mostly elusive, with ground-based imaging reliant on common contrast-enhancing methods (e.g., angular differential imaging) that can render such diffuse structures undetectable or photometrically suspect (Milli et al. 2012; Perrin et al. 2015, cf. their Figure 11).
Here we revisit the iconic HR 4796A debris system with new observations that focus on its exo-ring structures and environment revealed through deep Hubble Space Telescope (HST)/Space Telescope Imaging Spectrograph (STIS) six-roll PSF templatesubtracted coronagraphy (6R/PSFTSC) and simultaneous subtraction of the background starlight from its nearby M-star companion. This paper concentrates on the methodology and physical characterization of the debris system. In Section 2, we provide information on the stellar and disk components of the HR 4796 system and prior scattered-light imaging that revealed (only) its bright debris ring. Section 3 details the new HST/STIS observations and the observational paradigm using three STIS coronagraphic occulters and six field orientation angles enabling circumazimuthal image structure recovery over a very large stellocentric angle range. In Section 4, we present the end-to-end coronagraphic reduction and calibration processes, including multi-roll and dithered PSF template subtraction and multi-image combinations employed to produce a final "analysis-quality" (AQ) SB image. In Section 5, we compare the prior STIS 2001 and new 2015 epoch AQ imaging of the debris ring itself. The principal observational and metrical results (morphology, photometry, astrometry) for the extended debris structure, including the ring and its very large exo-ring halo as derived from the AQ image, are presented in Section 6. In Section 7, we discuss the HR 4796A/B system in the context of other exoplanetary debris disks with large exo-ring scattering structures, its M-star companion, and posited interaction with the local interstellar medium (ISM). In Section 8, we summarize key systemic attributes newly informed from this 
Table 1

HR 4796 System Components

\begin{tabular}{|c|c|c|c|c|c|c|c|c|}
\hline \multicolumn{9}{|c|}{ (A) Primary Star and Debris Disk } \\
\hline \multirow{2}{*}{ Target } & \multirow{2}{*}{$V_{\mathrm{mag}}^{\mathrm{a}}$} & \multirow[t]{2}{*}{$B-V^{\mathrm{a}}$} & \multirow[t]{2}{*}{ Spec. $^{\mathrm{b}}$} & \multirow{2}{*}{$\begin{array}{c}\text { Dist. }^{\mathrm{c}} \\
(\mathrm{pc})\end{array}$} & \multirow{2}{*}{$\begin{array}{l}\mathrm{Age}^{\mathrm{d}} \\
(\mathrm{Myr})\end{array}$} & \multirow{2}{*}{$\begin{array}{c}\text { Disk } \\
L_{\mathrm{IR}} / L_{\mathrm{star}}^{\mathrm{e}}\end{array}$} & \multicolumn{2}{|c|}{ Initial $H S T$ Disk Imaging } \\
\hline & & & & & & & Instrument & Reference \\
\hline HR 4796A & 5.774 & +0.012 & $\mathrm{~A} 0 \mathrm{~V}$ & 72.8 & $8 \pm 2$ & 0.0042 & NICMOS & Schneider et al. (1999) \\
\hline \multicolumn{9}{|c|}{ (B) M-Star Companion } \\
\hline Companion & Spec. $^{\mathrm{b}}$ & $V_{\text {mag }}{ }^{\mathrm{a}}$ & $\Delta \mathrm{Mag}^{\mathrm{a}}$ & Separation/P.A. ${ }^{\mathrm{f}}(2015)$ & References & & & \\
\hline HR 4796B & M2.5 & 13.3 & 7.5 & $7 ! .92 \pm 0 . .02 / 29.81$ & Jura (1995), Lagrange et al. (2012) & & & \\
\hline
\end{tabular}

Notes.

${ }^{\text {a }}$ From Hog et al. (2000) VizieR online catalog I/259.

${ }^{\mathrm{b}}$ From Houk (1982) VizieR online catalog III/80.

${ }^{c}$ Hipparcos parallactic distance from Van Leeuwen 2007 VizieR online catalog I/311.

${ }^{\mathrm{d}}$ Age estimation from Stauffer et al. (1995).

${ }^{\mathrm{e}}$ IR $(4 \mu \mathrm{m}-1 \mathrm{~mm})$ excess from Pascual et al. (2016).

${ }^{\mathrm{f}}$ As determined from this work with internal measurement uncertainty in P.A. \pm 0.04 and absolute uncertainty of the HST guide-star frame orientation $\approx \pm 0.1$.

investigation and offer some closing commentary on the future levering of $H S T /$ STIS PSFTSC.

\section{Targets}

In HST GO program 13786, we observed two 5-10 Myr A0V stars with IR and optically bright debris systems: HR 4796A (Table 1) and HD 141569A (Weinberger et al. 1999). Both stars have a priori well-known ringlike disks of starlightscattering material, and both possess early M-star companions (one for HR 4796A and two for HD 141569A). The HD 141569A system, in the context of our STIS observations, was discussed by Konishi et al. (2016). Herein, we report on the HR 4796A system, a member of the TW Hya association (TWA 11A), with key component characteristics given in Table 1.

The $\approx 76^{\circ}$ inclined ringlike debris disk of HR 4796A was initially imaged in 1998 by Schneider et al. (1999) in near-IR scattered light at 1.1 and $1.6 \mu \mathrm{m}$ with $H S T /$ NICMOS. These scattered-light discovery images were followed up with higherfidelity (but relatively shallow depth) observations in 2001 with HST/STIS two-roll coronagraphy in visible light (Schneider et al. 2009) that better revealed and detailed its steeply "edged" ringlike nature. Subsequent NICMOS observations (Debes et al. 2008) with multiband diagnostic filters suggested the possibility of radiationally evolved complex organic materials in the ring. As a bright, geometrically favorable debris disk target for episodically improving ground-based near-IR and narrow-field-angle adaptive optics (AO) technologies, the archetypical HR 4796A system was extensively observed with capability-driven focus on the debris ring itself, e.g., with Subaru/HiCIAO (Thalmann et al. 2011), VLT/NaCO (Lagrange et al. 2012), Gemini/NICI (Wahhaj et al. 2014), Gemini/GPI (Perrin et al. 2015), and Magellan/ MagAO (Rodigas 2015). Perrin et al. (2015) suggested that the GPI-detected polarized intensity signature of the debris ring may indicate that it is actually optically thick or partially self-shadowed on one side of the disk major axis. Ground-based observations of the debris ring by Thalmann et al. 2011 hinted at the possibility of some exo-ring materials in close external proximity to the ring ansa. This was suggested by Milli et al. (2012) as nonastrophysical in origin, potentially arising from artifacts resulting from angular differential imaging employed for contrast enhancement. However, prior to new STIS results discussed in this paper, a posited
HR 4796A exo-ring scattered-light halo had been essentially unexplored.

\section{New STIS Observations}

HR 4796A (discussed herein) and HD 141569A (Konishi et al. 2016) are A0/B9-type stellar hosts to two of five exoplanetary debris systems imaged with deep STIS 6R/ PSFTSC in HST GO program 13786 (PI: G. Schneider); see Sch16. This program also included three ringlike debris systems with older solar analog (G-star) hosts (HD 207129, HD 202628, and HD 202917). In all cases, we very closely followed the observational paradigm detailed in Sch14 that used two coronagraphic image-plane occulters of different angular widths and exposure time depths. Together, these provide very large stellocentric angle field coverage (in principle, in the range $\sim 0$ !' $2 \leqslant r \leqslant \sim 15^{\prime \prime}$ ) at high contrast (cf. Sch14, their Figure 27) and a large imaging dynamic range $\left(>10^{5}\right.$ in the final image combination after starlight suppression via PSF subtraction).

Background discussion of the observational design to minimize PSF-subtraction residuals while enhancing image contrast, leading to the observation plan for HR 4796A and its PSF star HR 4735 as summarized in Table 2, are given in Sch14, Sch16, and Schneider et al. (2017). Herein, HR 4796A is observed in a total of six HST orbits at different field orientation angles in two sets of three contiguous visits, each interleaved with a single-orbit visit of its PSF template star. Multiple exposures with HR 4796A or HR 4735, as sequentially occulted by the STIS BAR5 and WedgeA-1.0 masks (for narrow fieldangle and deep wide-field coronagraphy, respectively), were obtained to fill each target visibility period; see Table 2 (following Sch14). In two HR 4796A visits, STIS WedgeB was used to simultaneously occult HR 4796B.

The STIS 50CCD instrumental configuration employed for all observations provides a broad visible-light passband with a pivot wavelength of $0.575 \mu \mathrm{m}$ and FWHM of $0.433 \mu \mathrm{m}$. The image scale is 50.77 mas pixel $^{-1}$, sampling the PSF with a diffractionlimited resel of 72 mas. ${ }^{7}$ With multi-roll combination, critical (Nyquist $Q=2$ ) or better sampling is achieved in

\footnotetext{
STIS employs a Lyot stop with outer radius 0.835 of the telescope pupil for an effective diameter of $2.00 \mathrm{~m}$.
} 
Table 2

Observation/Exposure and Data Log

\begin{tabular}{|c|c|c|c|c|c|c|c|}
\hline $\begin{array}{l}\text { Target } \\
\text { (Disk/PSF) }\end{array}$ & $\begin{array}{l}\text { UT Date } \\
\text { Obs. Start }\end{array}$ & $\begin{array}{l}\text { Orientat }^{\mathrm{a}} \\
(\mathrm{deg})\end{array}$ & $\begin{array}{l}\text { BAR5 }^{\text {b }} \\
\text { \#Exp. }\end{array}$ & $\begin{array}{l}\text { BAR5 } T_{\mathrm{EXP}} \\
\text { All Visits (s) }\end{array}$ & $\begin{array}{l}\text { W1.0 } \\
\text { \#Exp. }\end{array}$ & $\begin{array}{c}\mathrm{W} 1.0 T_{\mathrm{EXP}} \\
\text { All Visits (s) }\end{array}$ & $\begin{array}{c}\text { Visit Data } \\
\text { ID }^{\mathrm{c}}\end{array}$ \\
\hline HR 4796A & 2015 Jan 20 & $232.6,254.9^{\mathrm{d}}, 276.9$ & 72 & 129.6 & 33 & 4633.2 & $41,42,44^{\mathrm{e}}$ \\
\hline HR 4735 & 2015 Jan 20 & 255.1 & 24 & 36.0 & 13 & 1502.8 & 43 \\
\hline HR 4735 & $2015 \mathrm{Jul} 09$ & 70.0 & 24 & 36.0 & 13 & 1502.8 & 47 \\
\hline
\end{tabular}

Notes.

a Orientat: In the Science Instrument Aperture File (SIAF); measured from image $+\mathrm{Y}$ axis CCW to celestial north.

${ }^{\mathrm{b}}$ Including $+1 / 4,0$, and $-1 / 4$ pixel ( \pm 12.7 mas) cross-centerline BAR5 dithers (see Schneider et al. 2017).

${ }^{c}$ Visit-level data set ID as assigned by MAST. GO 13786 data archived as ocjc + Visit_Data_ID + *.

${ }^{\mathrm{d}}$ Second of three target visits in each set scheduled at nominal roll (first and third target visits at off-nominal roll).

${ }^{\mathrm{e}}$ For W1.0 imaging, in visits 44 and 45, respectively, HR 4796B itself is occulted by Wedges B and A; see Figure 3.

Table 3

Contemporaneously Interleaved, Color-matched PSF Template Star

\begin{tabular}{|c|c|c|c|c|c|c|c|c|}
\hline \multirow{2}{*}{$\begin{array}{l}\text { Disk } \\
\text { Target }\end{array}$} & \multirow{2}{*}{$\begin{array}{l}\text { PSF } \\
\text { Star }\end{array}$} & \multirow{2}{*}{$\begin{array}{l}\text { PSF } \\
\text { Spec }^{\text {a }}\end{array}$} & \multirow{2}{*}{$\begin{array}{l}\text { PSF } \\
\mathrm{Vb}\end{array}$} & \multirow{2}{*}{$\begin{array}{c}\mathrm{PSF} \\
B-V^{\mathrm{b}}\end{array}$} & \multirow[t]{2}{*}{$\Delta[B-V]^{\mathrm{c}}$} & \multirow{2}{*}{$\begin{array}{c}\text { Target } \rightarrow \text { PSF } \\
\text { Slew Distance }\end{array}$} & \multicolumn{2}{|c|}{ PSF } \\
\hline & & & & & & & & \\
\hline HR 4796A & HR 4735 & B9V & 5.558 & +0.007 & +0.005 & $7: 3$ & -0.2 & -2.0 \\
\hline
\end{tabular}

Notes.

${ }^{a}$ From Houk (1982) VizieR online catalog III/80.

${ }^{\mathrm{b}}$ From Hog et al. (2000) VizieR online catalog $\mathrm{I} / 259$.

c $\Delta[B-V]$ : Difference in $[B-V]$ color index for disk host and PSF template stars.

d $\triangle$ ONR: Difference in off-nominal roll angle for the two epochs of template observations.

most pixels. Additional instrumental information on the STIS coronagraph and its image-plane occulters may be found in Grady et al. (2003), Riley (2017), ${ }^{8}$ and Schneider et al. (2017).

For HR 4796A, two details in the observational strategy differed from the GO 13786 solar analog targets. (1) Given the a priori known small angular size of the debris ring itself $(r=1$ !" 05$)$, for intra- and endo-ring imaging, we made use of the 0 ". 15 half-width BAR5 occulter, rather than WedgeA at its 0"6 full-width position, as we also did for HD 141569A (Konishi et al. 2016). (2) For exo-ring imaging, in two of six roll angles employed (in visits numbered 44 and 45), while HR 4796A was occulted at the WedgeA-1.0 position, HR 4796B was simultaneously occulted elsewhere along the orthogonal STIS B and A wedges, respectively, to improve coronagraphic contrast in the exo-ring portions of the debris system toward its nearby M-star companion. With these absolute orientation constraints, the visit-contemporaneous BAR5 field orientations were nonoptimal ${ }^{9}$ but acceptable with respect to both the smallest possible circumazimuthal inner working angles (IWAs) and sampling diversity in field rotation. This was a compromise deemed acceptable in observation planning to mitigate the cost, otherwise, of additional HST orbits, where the observational focus was on the exo-ring region.

Due to $H S T$ roll-range limitations at any epoch, 6R/PSFTSC observations were scheduled in two sets of three target (plus one interleaved PSF) single-orbit visits half a year apart. The intra-epochal visits were incrementally rolled about the occulted target location by $\approx 22^{\circ} .5$ (the half-angle between the STIS occulting wedges and Optical Telescope Assembly

\footnotetext{
8 http://www.stsci.edu/hst/stis/documents/handbooks/currentIHB/stis_ ihb.pdf

9 The long axis of the BAR5 occulter is rotated $\approx 72^{\circ}$ counterclockwise in the instrument frame with respect to WedgeA. The SIAF orientation of the latter was used to define the visit-level spacecraft orientation requirements.
}

(OTA) diffraction spikes). See the Table 2 column labeled Orientat for the resulting field orientations as executed.

For the shorter-exposure BAR5 images of both the disk and PSF template targets, we performed three-point linear "crossbar" dithers of $[-0.25,0.00,+0.25]$ pixels $( \pm 12.7$ mas $)$ as a precaution against a priori known possible target acquisition imprecision in initial target placement with respect to the midline of the BAR5 occulter. Table 2, following Sch16, gives the observational details for both the HR 4796A and PSF template star observations, with total (six-roll combined) integration times achieved of $\approx 9.3 \mathrm{ks}$ in most pixels for the deep WedgeA-1.0 imaging of the debris system. Further details of the observation plan are available from STScI. ${ }^{10}$

\section{Data Reduction and Processing}

\subsection{Basic Image Calibration and Reduction and Host-star PSF Subtraction}

To produce AQ, photometrically calibrated, count-rate images with the underlying stellar host PSF removed, we exactly followed the methodology and procedures as detailed in Sch14 and Sch16, to which we refer the reader for details. For PSF subtraction, we used an observationally interleaved, disk target-specific PSF template star; see Table 3. Based on prior demonstrated performance, a PSF template star was chosen that (a) had optical color identically matched $(|\Delta[B-V]|<0.01)$, (b) was located within $10^{\circ}$ on sky, and (c) was contemporaneously observable at both epochs with $<5^{\circ}$ difference in nominal roll with respect to the HR 4796A visit immediately preceding. Coronagraphic PSF template images employed for primary star subtraction were reduced from intra-visit combined FLT (instrumentally calibrated, exposure level) images

\footnotetext{
${ }^{10}$ http://www.stsci.edu/hst/phase2-public/13786.pro
} 


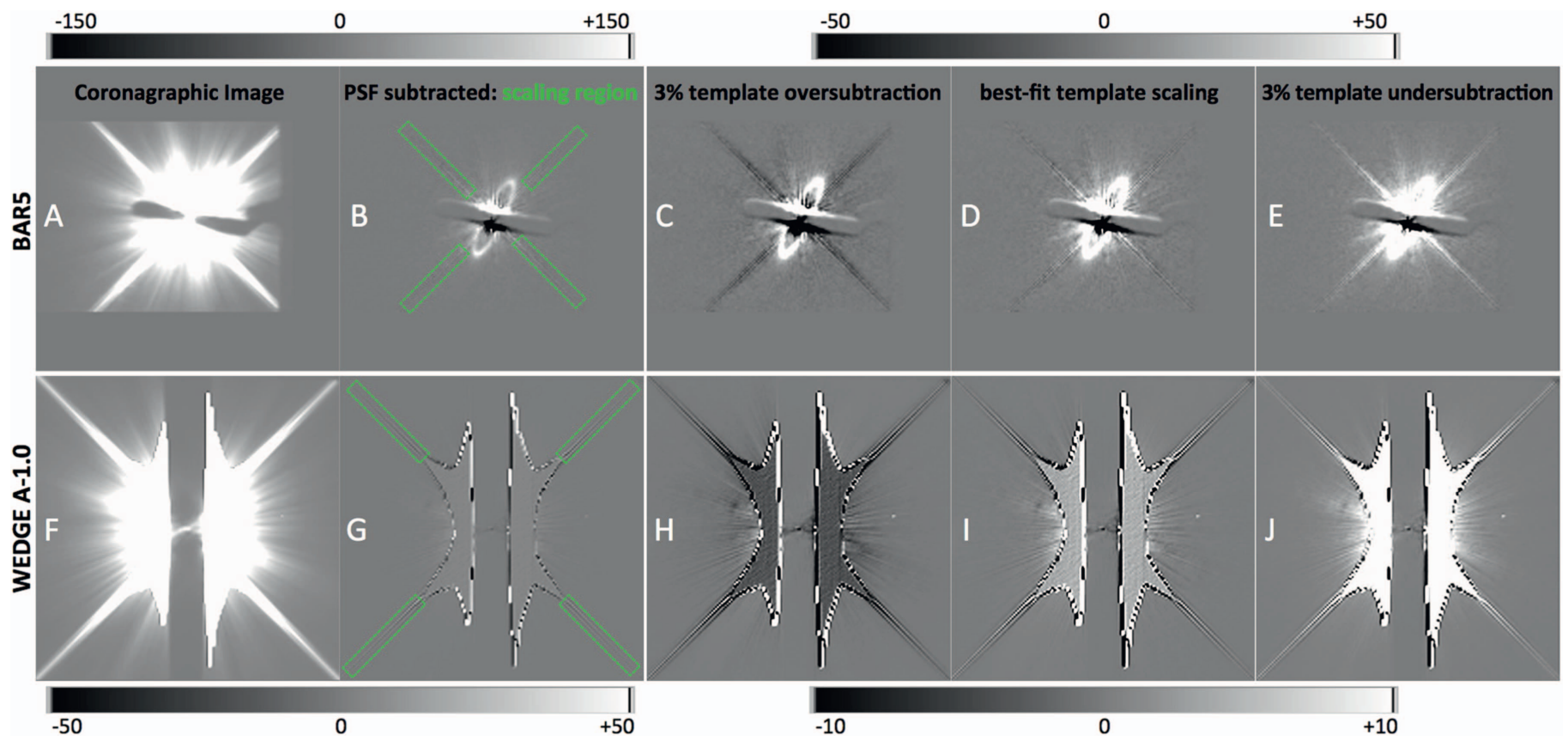

Figure 1. Panels (A) and (F): representative HR 4796A coronagraphic images with the STIS BAR5 and WedgeA-1.0 occulters prior to PSF template subtraction Panels (B) and (G): corresponding images after PSF subtraction minimizing residuals along the diffraction spikes in the green outlined regions. Panels (C)-(E) and (H)-(J) demonstrate the ability to ascertain to high precision the target-to-template PSF brightness ratio for PSF subtraction by closely nulling the flux along the HST diffraction spikes (see main text). All panels are at the same celestial orientation (Orientat $=232.6$ ) and image scale (field: $\left.8^{\prime \prime} .1 \times 8^{\prime \prime} .1\right)$ and have equal-amplitude plus-to-minus linear stretches about zero (see gray-scale intensity bars in counts s ${ }^{-1}$ pixel $^{-1}$ ) to illustrate both subtraction residuals imposed on the disk signal with PSF subtractions. Panels (B) and (G) have the same data as panels (D) and (I), stretched to levels to best illustrate the ring in panel (B) and diffraction spike nulling in panel (D). A deeper stretch is needed to reveal the starlight-scattering exo-ring material just hinted at beyond the central region of image saturation in panel (I) (see Figure 2).

produced with STScI's calstis software in a manner identical to that of the disk host images themselves, then converted to instrumental count-rate units (counts s${ }^{-1}$ pixel $^{-1}$ ).

The contemporaneous HR 4735 PSF BAR5 and WedgeA1.0 coronagraphic template images from visits 43 and 47 were astrometrically coregistered and iteratively intensity scaled to simultaneously match the position and brightness of the corresponding HR 4796A stellar PSFs in all target images from corresponding visits 41,42 , and 44 and 45, 46, and 48, respectively. We treated the template brightness and $\mathrm{X} / \mathrm{Y}$ (SIAF) image position as free parameters to minimize PSFsubtraction residuals along the unocculted primary star diffraction spikes per Sch14. See Figure 1, a representative example from visit 41 with the same celestial image orientation and spatial scale for the BAR5 (panels (A)-(E)) and WedgeA1.0 (panels $(\mathrm{F})-(\mathrm{J})$ ) observations illustrated.

Panel (A) of Figure 1 is a BAR5 coronagraphic image of HR 4796A with the PSF halo incompletely suppressed and (on the diagonals) contains the unapodized $H S T$ diffraction spikes. Panel (A) is shown at the same display stretch maximum as the peak brightness of the debris ring that is revealed after PSF subtraction in panel (B). In panel (B), at that level, the diffraction spike residuals are barely visible. The green rectangular areas in panel (B) enclose the regions of the diffraction spikes used to minimize the PSF-subtraction residuals. The instrumental brightening/darkening along the BAR5 edges, in diametric opposition to the location of coregistered target and template stars, results from target acquisition positioning imperfections (differential decentration) with respect to the BAR5 midline (see Section 4.3).
To demonstrate that the correct intensity scaling was found by variance minimization for the template PSF (Sch14), we forced small $( \pm 3 \%)$ parametric variations about the best-fit target-to-template 50CCD band brightness ratio, $\mathrm{x} 0.811$ (panels (C) and (E)). The oversubtractions and undersubtractions at this level are obvious when compared with the best-fit panel (D) (restretched the same as panels (C) and (E) to best illustrate but otherwise identical to panel (B)).

Panels (F)-(J) are similar to panels (A)-(E) but for WedgeA1.0. By observational design, to enable deep imaging at larger stellocentric angles, these images saturate the detector in the near-stellar central region beyond occulting WedgeA, obscuring the debris ring itself that is separately imaged (unsaturated) using the BAR5 occulter.

A robust and well-determined target-to-template flux-density ratio was independently established from a suite of $60 \mathrm{PSF}-$ subtracted images. These images were derived from all six visits (each at a different field orientation) in two epochs: six visit-level images for WEDGEA-1.0 (Figure 3) and 54 dither combinations for BAR5 (Figure 6). A dispersion of $<1 \%$ from the average flux-density scaling ratio of $\mathrm{x} 0.811$ among all images was found and adopted. No statistically significant bias was found between the BAR5 and WEDGEA-1.0 images in the spatial regions commonly sampled. See Sch14 for additional details of the PSF-subtraction method and further discussion of constraints upon PSF template star selection and observations as reflected in Table 3.

\subsection{WedgeA-1.0 Companion PSF Subtraction}

For the HR 4796 system, primary-only PSF subtraction leaves the sky background in the direction toward the M-star 


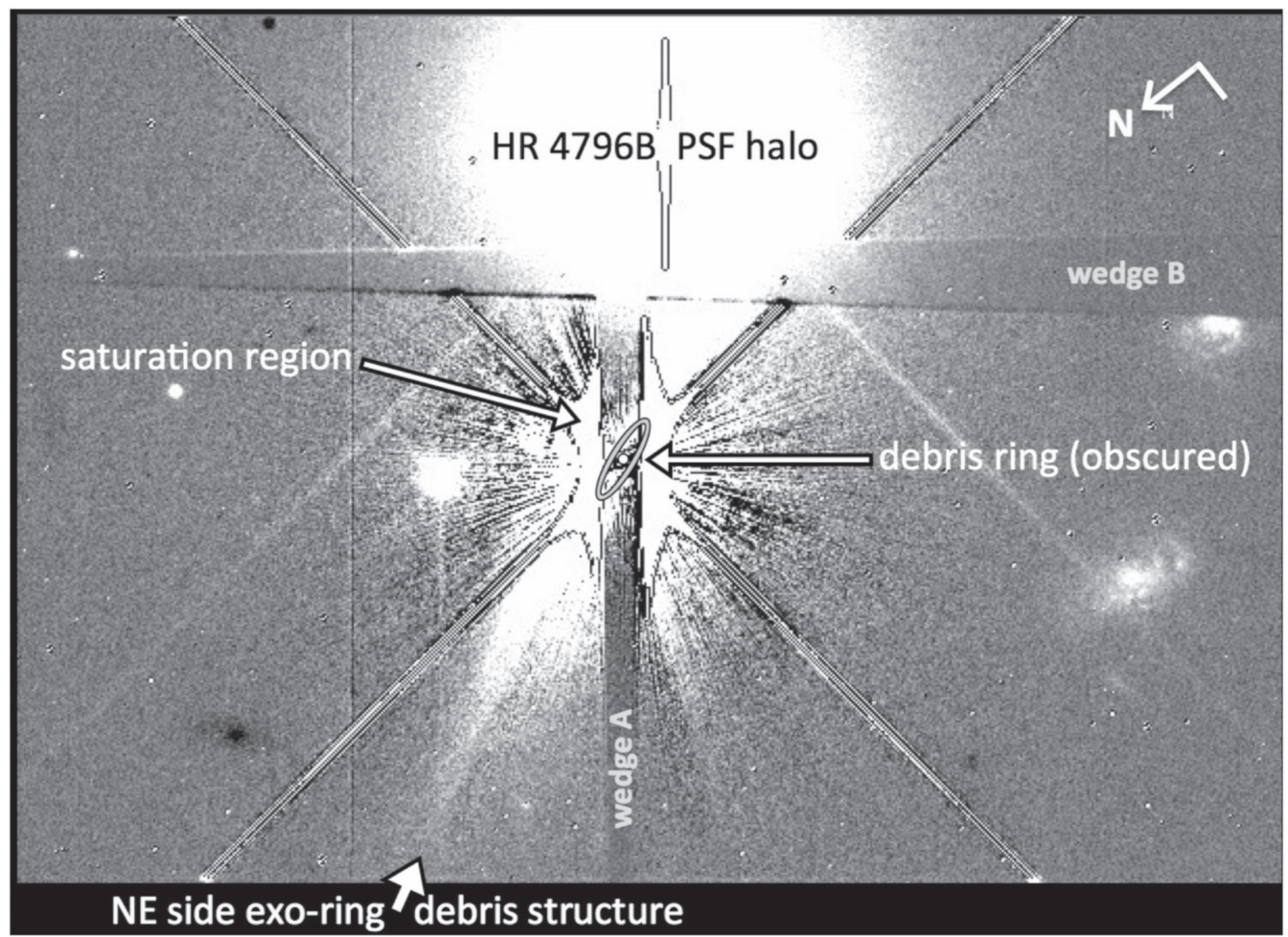

Figure 2. HR 4796A, visit 41, WedgeA-1.0 occulter. After visit-level primary PSF template subtraction, the northeast half of a large exo-ring scattering structure is revealed, but the diametrically opposed southwest part of the debris system is obscured by stellar light from the M-star companion's PSF halo. Linear display stretch from -0.05 to +0.1 counts s $^{-1}$ pixel $^{-1}$. FOV: 30 !' $9 \times 211^{\prime \prime} 2$. The various features and components identified in this figure also appear in the unannotated panels in Figures 3 and 5.

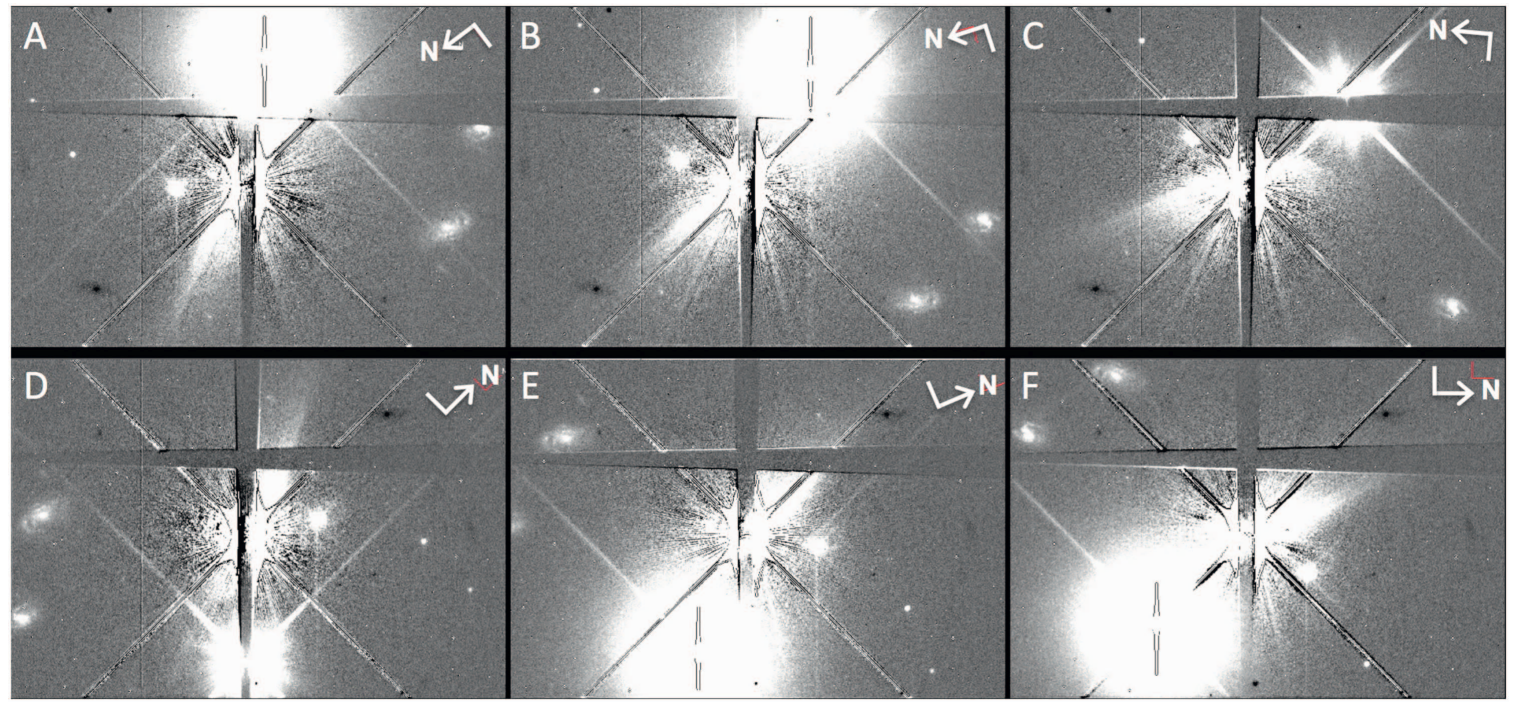

Figure 3. HR 4796A PSF-subtracted images at all six celestial orientation angles (see Table 2). Panels (A)-(C): visits 41, 42, and 44. Panels (D)-(F): visits 45, 46, and 48. In panels (C) and (D) (visits 44 and 45), HR 4796B is obscured by STIS wedges (B) and (A), respectively, resulting in diminished intensity in the companion PSF halo and unveiling the southwest part of the debris system prior to further suppression with PSF template subtraction. While from image to image, the celestial field in the SIAF frame rotates about HR 4796A, the STIS wedges and OTA diffraction spikes are rotationally invariant. See Figure 2 as a guide (identical to panel (A) above) to the major astrophysical and instrumental components in these images. All panels with a linear display stretch from -0.05 to $+0.1 \mathrm{counts} \mathrm{s}^{-1}$ pixel ${ }^{-1}$ and FOV of 30 " $9 \times 21 "$. 2 .

companion, beyond the bright inner-disk regions of its stellar host, polluted with light from the companion PSF halo in the deep WedgeA-1.0 imaging. For example, see Figure 2, illustrating in detail a deep host-star-only PSF-subtracted image of the HR 4796A system from representative visit 41 (same data as in Figure 1). In Figure 3, compare the background astronomical field at all six observed celestial orientation angles without (panels (A), (B), (E), and (F)) and with (panels (C) and (D)) simultaneous companion coronagraphic obscuration. The latter two cases enable the visibility of the southwest side of the debris structure even before companion PSF subtraction further improves its detectability.

To mitigate the unocculted companion background contamination with sufficiency compared to the level of the sky 


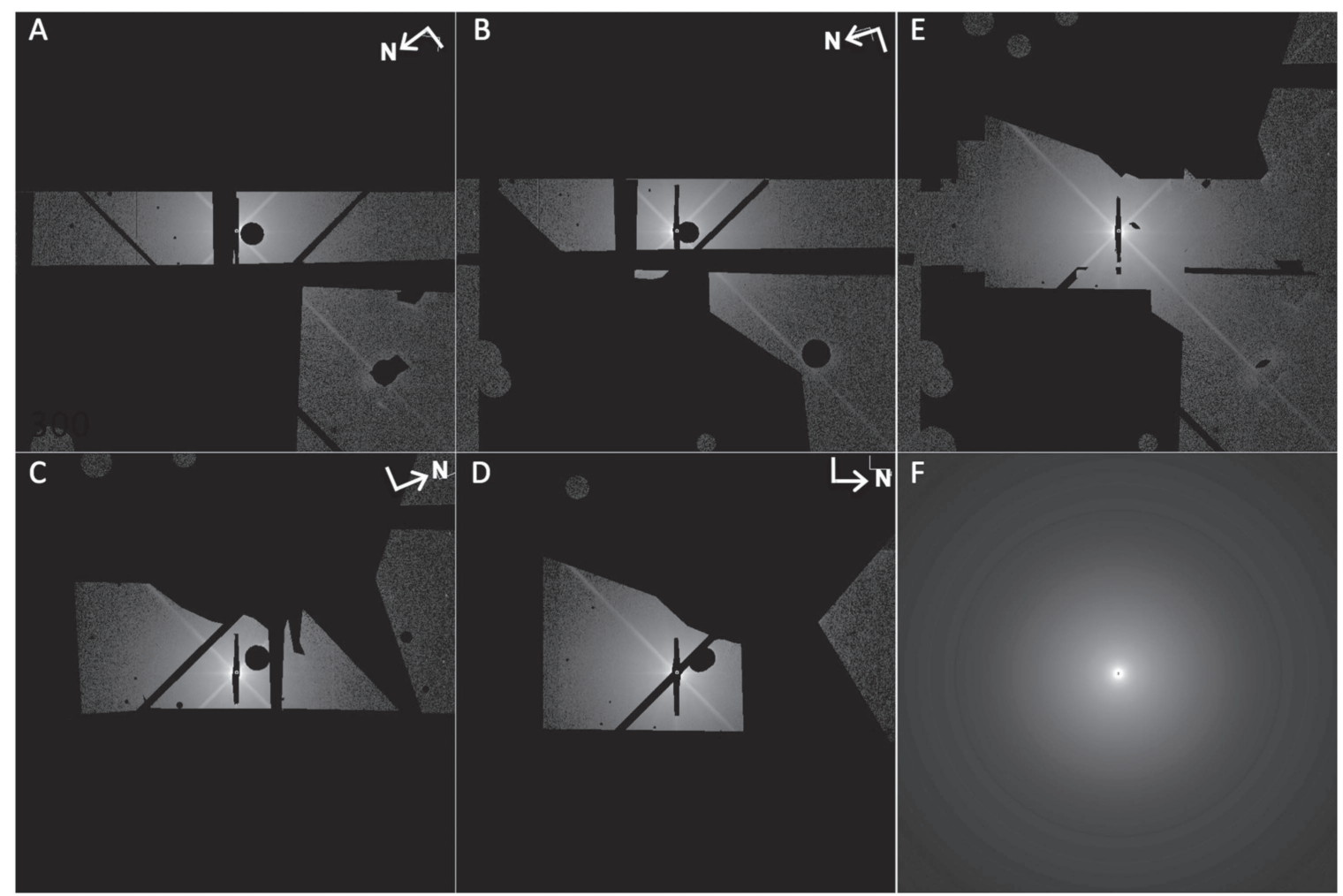

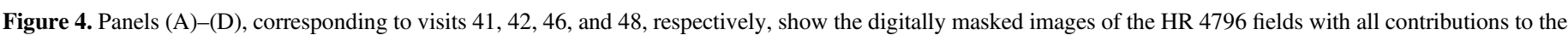

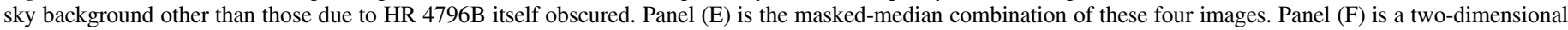

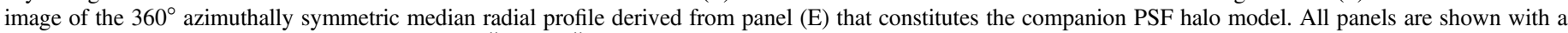
$\log _{10}$ display stretch. The FOV of all panels is 33 !! $2 \times 33$ ! 2 .

background beyond the detectable periphery of the circumstellar debris system, we similarly subtracted a model of the M2.5V companion PSF halo. This model was empirically derived from astrometrically coregistered observations of HR 4796B in host-star-subtracted visit-level images 41, 42, 46, and 48 after digitally masking all other astronomical sources (background stars, galaxies, the HR 4796A disk) and HST/ STIS image artifacts (OTA diffraction spikes, STIS occulting wedges, image ghosts, saturated pixels) in the field; see Figure 4. After masked-median combination (Figure 4, panel (E)), a $360^{\circ}$ azimuthal median profile about the four-visit combined HR 4796B image was used to produce a first-order azimuthally symmetric extended PSF halo model (Figure 4, panel $(\mathrm{F})$ ) used for unocculted companion PSF subtraction.

The fine structure of the PSF is somewhat different in detail in coronagraphically occulted images (other than just in intensity), i.e., in visits 44 and 45 for HR 4796B. This, however, is of significance only within a few arcseconds of the companion that is beyond the periphery of the HR 4796A debris system (see Figure 5, after PSF subtraction). Nonetheless, for these visits (only), we used a weighted linear combination of a Tiny Tim model PSF (Krist et al. 2011) and the empirical PSF model described above. The diameter of the Tiny Tim PSF is limited by its optical model to $r \leqslant 9^{\prime \prime}$ but was seen to smoothly transition at this radial distance to the empirical halo model with no significant discontinuity. While the Tiny Tim PSF model does not replicate with high fidelity the coronagraphic PSF fine structure close to the wedge edges (but unnecessary for this purpose), the observed companion PSF halo at larger distances (superimposed upon the outer reaches of the debris system) in combination is well reproduced.

Figure 5 illustrates all six HR 4796A and B PSF-subtracted images (before multi-roll combination) wherein the full extent of the asymmetric exo-ring debris system is reproducibly revealed in all commonly sampled regions, corotating with the telescope in the SIAF frame. An examination of Figure 5 (after subtraction of the HR 4796B halo model) reveals a prominent image ghost in the unobscured companion images that is not remediated by the PSF halo model. However, this too only appears within a few arcseconds of the companion and does not contribute to the residual background in the outer reaches of the HR 4796A debris system.

\subsection{BAR5 Primary PSF Subtraction}

The shorter BAR5 exposures were designed to produce high signal-to-noise ratio $(\mathrm{S} / \mathrm{N})$ images at small stellocentric angles $\left(r \lesssim 2^{\prime \prime}\right)$ to the effective IWA limit without saturation. Excess background light from the $\approx 8^{\prime \prime}$ distant M-star companion in this region is not significant, so companion PSF subtraction is unnecessary. However, target acquisition position offsets in the relative placement of the disk and template targets, if sufficiently large, can result in differential signal gradients near the bar edges. This is partially mitigatable with target and template small- (0.25 pixel) subpixel-dithered imaging as executed. For each BAR5 disk target visit, we create a suite of nine PSF-subtracted images (54 in all with six rolls) of all target and template dither permutations, otherwise following the same process for PSF subtraction as previously described. For example, see Figure 6, with all images equally stretched linearly about zero to illustrate BAR5-edge artifacts (hard black 

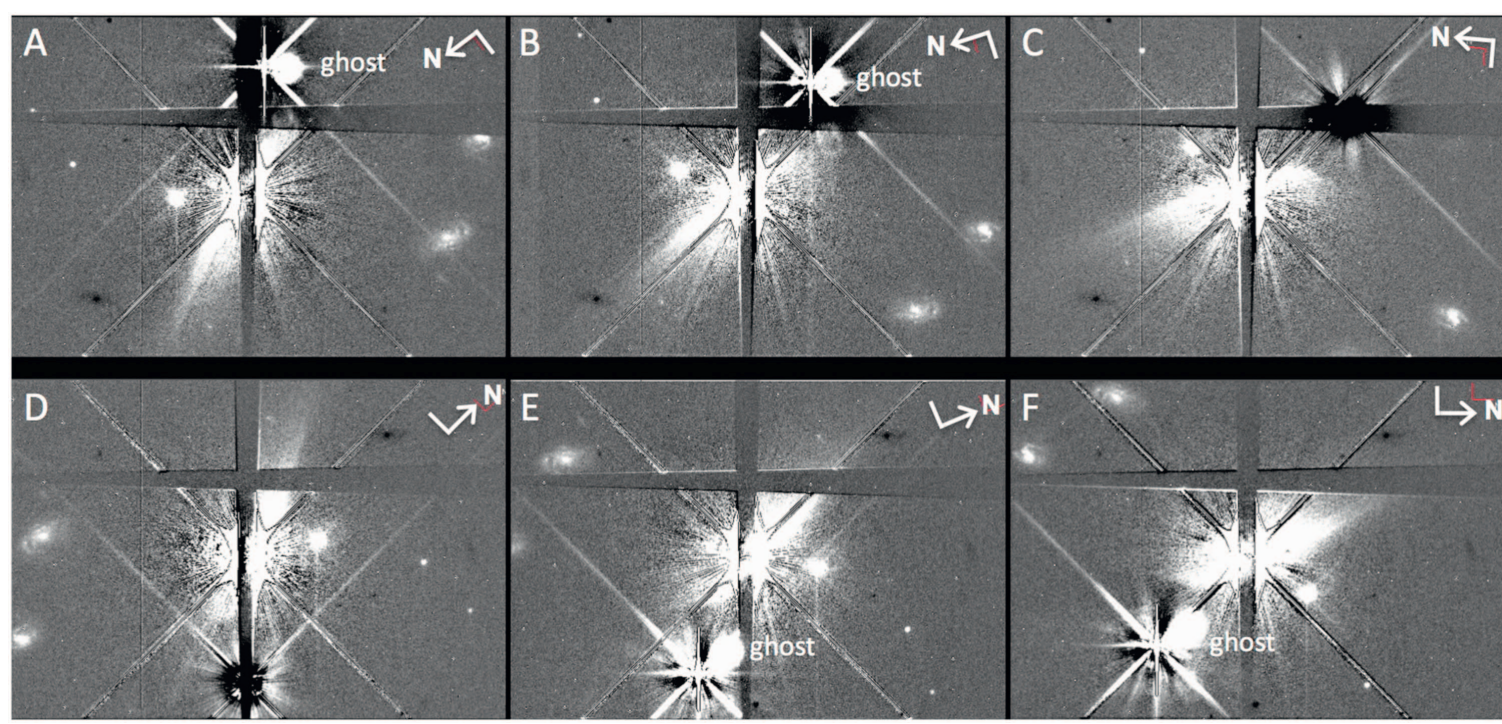

Figure 5. After subtraction of a PSF halo model for HR 4796B, the asymmetric debris system beyond the central saturation region is fully revealed with each field reorientation. From frame to frame, the primary star's saturation region, diffraction spikes, and STIS occulting wedges remain fixed in image-plane location as the telescope is reoriented about the primary star, in combination providing complete $360^{\circ}$ mapping of the outer debris system. Presented identically as in Figure 3 with panels (A)-(C) showing visits 41, 42, and 44 and panels (D)-(F) showing visits 45, 46, and 48.

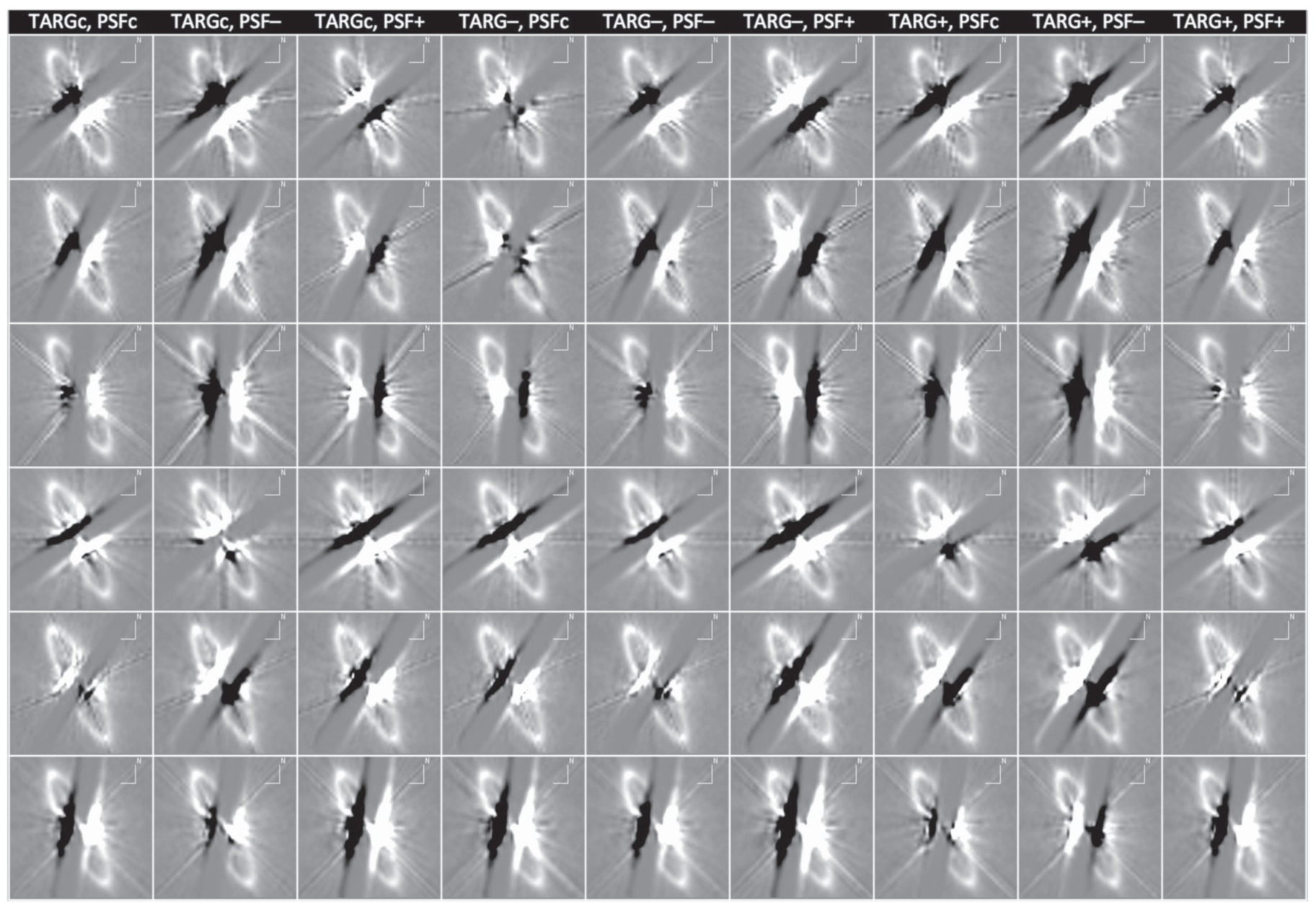

Figure 6. BAR5 cross-bar-dithered PSF-subtracted imaging illustrating all nine dither offset permutations ("c" = centered, "-" = minus, "+" = plus) for targetminus-PSF template subtractions for each target visit (top down: visits 41, 42, 44, 45, 46, and 48). All images are rotated with north up (east to the left) and stretched to illustrate BAR5-edge artifacts and subtraction residuals. Affected regions are individually masked prior to multi-image median combination. The FOV of each panel is 2 ". $46 \times 2$ "! 46. See Table 2 for SIAF celestial orientations. 


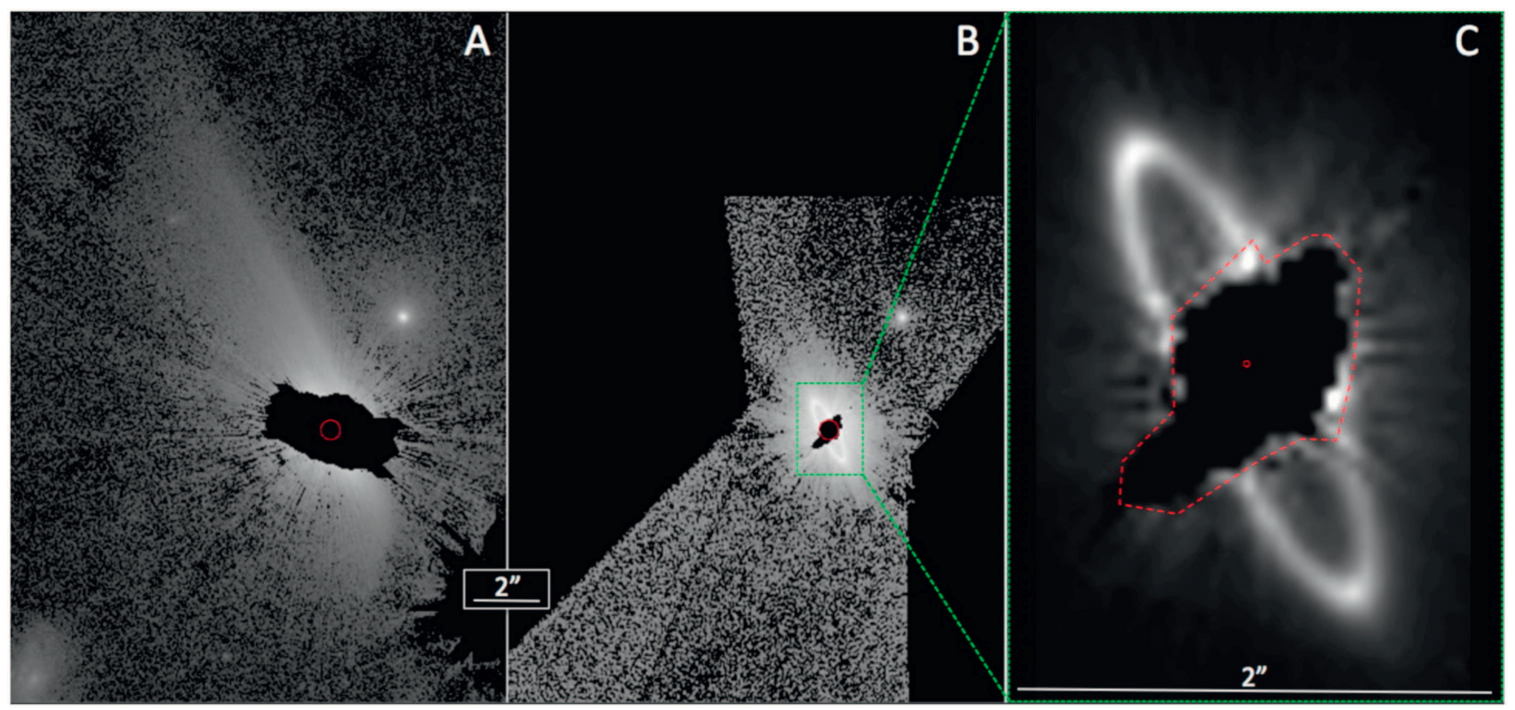

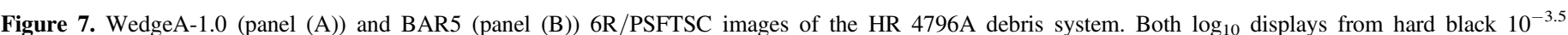

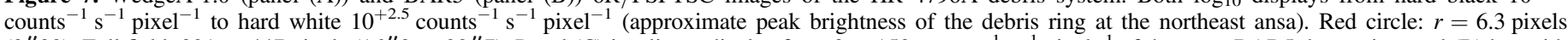

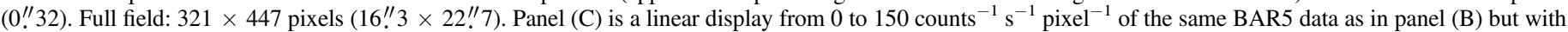
a focus on the debris ring itself $(\mathrm{FOV}=2 ! .9 \times 2$." 1$)$. All images are north up, east to the left.

and hard white) that are later masked (rejected) prior to multiimage combination. For further details of the process, see Schneider et al. (2017). For HR 4796A, the six-roll space in celestial orientation was not optimally tiled to best sample fully around the debris ring due to the absolute orientation constraints imposed for the contemporaneous WedgeA-1.0 imaging required therein to simultaneously occult HR 4796B. This resulted in a conservative effective IWA of $\approx 0$ "! 32 in the BAR5 PSF-subtracted imaging, larger than the physical angular width of the occulting mask itself.

\subsection{Six-roll Image Combinations}

All PSF-subtracted images were coaligned on the astrometrically determined location of the occulted host star using the "X-marks-the-spot" method (Sch14) and rotated about the star to a common (north up) orientation.

(1) WEDGEA-1.0: The six visit-level PSF-subtracted countrate images, deeply exposed with WedgeA-1.0, were median combined after digitally masking pixels unsampled or affected by the STIS occulting wedges, image saturation, stellar diffraction spikes, or in the close presence of HR 4796B. The fully reduced $9.26 \mathrm{ks}$ 6R/PSFTSC WedgeA-1.0 image, inclusive of all unmasked data, is shown in Figure 7(A). The central region (in black) remains unsampled and obscures the debris ring that was contemporaneously separately imaged with the BAR5 occulter.

(2) BAR5: The nine subpixel-dithered PSF-subtracted images of HR 4796A using the BAR5 occulter (designed to image the debris ring itself) in each of the six visits, i.e., 54 images in total, were similarly median combined using separate digital masks for each image. The image-specific BAR5 digital masks reject image artifacts as described above for WedgeA1.0, as well as pixels along and adjacent to the BAR5 edges brightened (positive) or dimmed (negative) with diametrically opposing parity due to imperfect target acquisition placement. Figure 6 illustrates the diversity in such images before digital masking. The fully reduced 6R/PSFTSC BAR5 image is shown in Figure $7(\mathrm{~B})$ at the same image scale and orientation as the WedgeA-1.0 image (Figure 7(A)).
The small red circles in panels (A) and (B) indicate the approximate effective IWA of $r=0$ ". 32 achieved along the debris ring major axis using the BAR5 occulter. The effective IWA varies azimuthally around the star. The dashed red polygon in panel (C), with a focus on the debris ring itself, indicates the region of unsampled, or bar-edge degraded, pixels remaining in the multi-roll combined BAR5 image.

\subsection{AQ Data Image}

The separately reduced 6R/PSFTSC Wedge A-1.0 and BAR5 images were then merged by replacing pixels in the central void region of the WedgeA-1.0 image (Figure 7(A)) with those well-exposed in the BAR5 image (from Figure 7(B)). Before merging in this manner, the spatially overlapping WedgeA-1.0 and BAR5 image pixels beyond the WedgeA-1.0 saturation region were tested for intensity biases in commonly sampled areas that can arise from differential charge transfer inefficiency effects (see Debes et al. 2017) and found, on average, $<|1 \%-2 \%|$ pixel $^{-1}$ with some azimuthal dependence. This AQ data image, as presented in Figure 8 and derived in this manner, is the basis for the metrical analysis and subsequent discussions in the remainder of this paper.

\subsection{Instrumental Sensitivity and Absolute Photometric Calibration}

Throughout this paper, we present observational photometric results in instrumental count rates based on the AQ image shown in Figure 8. To convert to physical units of 50CCD spectral-band $(0.58 \mu \mathrm{m}) \mathrm{SB}$ and flux density, we adopt the STIS instrumental sensitivity and absolute photometric calibration as discussed in Schneider et al. (2016; their Section 5). For an instrumental configuration with $\mathrm{CCD}$ GAIN $=4$, as used for all observations presented herein, this gives rise to 1 count s${ }^{-1}$ pixel $^{-1}=4.55 \times 10^{-7} \mathrm{Jy}$ or $177 \mathrm{mJy} \mathrm{arcsec}^{-2}$ $\left(=18.04 V_{\text {mag }} \operatorname{arcsec}^{-2}\right)$. 


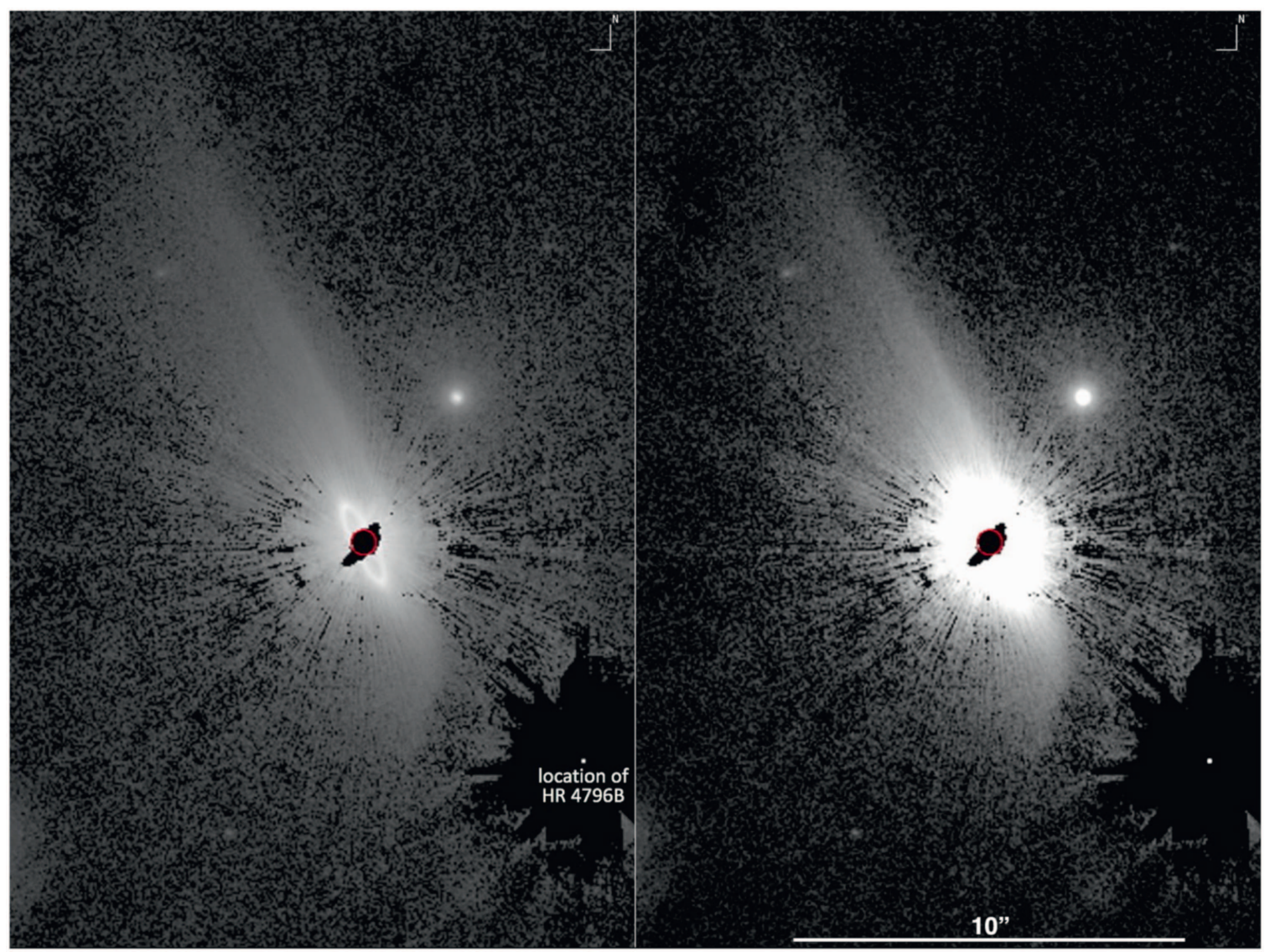

Figure 8. WedgeA-1.0 deep plus BAR5 shallow AQ imaging in a 16" (315 pixels) $\times 24^{\prime \prime}$ (472 pixels) region of the HD 4796A multi-roll FOV reveals the full extent, architecture, and morphology of the debris system from $r=0$ ". 32 (red circle centered on the coronagraphically occulted HR 4796A) outward. Left: $\log _{10}$ display stretch from [-3.5] (hard black) to [+2.5] dex counts s ${ }^{-1}$ pixel $^{-1}$ (hard white, $2 \mathrm{x}$ brighter than the peak brightness of the northeast ansa of the debris ring). Right: same data displayed from $[-2.5]$ to $[+0.5]$ dex counts s ${ }^{-1}$ pixel $^{-1}$ while obscuring the brighter debris ring itself by display saturation better details some of the structure and features within the exo-ring outer regions of the debris system. In both display images, the white dot in the southwest corner of the frame is the location of the digitally masked and simultaneously PSF-subtracted M2.5V companion HR 4796B.

\section{Comparison of 2001 and 2015 EPOCH Debris Ring Images}

Our GO 13786 (epoch 2015) 6R/PSFTSC imaging of the HR 4796A debris ring improves upon the fidelity of the prior (epoch 2001) two-roll-only STIS image from HST program 8624 discussed by Schneider et al. (2009; reproduced in Figure 9(A) from the published data) for several reasons. First, the combined use of the BAR5 (over Wedge A) occulter with multiple azimuthally well-tiled orientations enabled both a smaller effective IWA and a much smaller area beyond the IWA otherwise obscured by the occulter (compare the central uniform gray areas in Figures 9(A) and (B)). Second, the use of only a single roll differential $\left(\Delta \mathrm{roll}=15^{\circ}\right)$ in GO 8624 precluded much more efficacious decorrelation of quasi-static PSF-subtraction residuals achieved with the multi-roll visits in GO 13786. Compare, e.g., the "clumpiness" due to smallspatial-scale image artifacts (quasi-stable in the instrument aperture frame that corotates with the telescope) along the epoch 2001 debris ring image, in particular to the southwest of the star, that are largely absent in the 2015 image. Third, the use of cross-BAR5 subpixel dithers in the 2015 data set further improves on reducing PSF-subtraction artifacts, as well as occulter-edge effects near the star due to target-to-template target acquisition placement imperfections (Figure 6). For example, note the radial structures (image artifacts) interior to and traversing the debris ring that are particularly prominent to the southwest of the star in the 2001 image in Figure 9(A).

Additionally, the PSF star used in the GO 8624 observations, HR 4748, was chosen with angular proximity (very small spacecraft slew) as the highest priority in selection due (then) to programmatic constraints. With $\Delta[B-V]=+0.075$ as a PSF template, while not ill-suited for lower contrast imaging, HR 4748 is not as good a color match to HR 4796A as HR 4735 $(\Delta[B-V]=+0.005)$ that we adopted for our 2015 observations. The prior chosen PSF template star provided a globally optimized 2001 epoch image of the debris ring (Figure 9(A)) but resulted in some local chromatic oversubtractions of the stellar light within $\sim 1^{\prime \prime}$ of the star; see Schneider et al. (2009; their Section 3.3). This is apparent in Figure 9(A) with contiguous areas of flux density $<0$ count s$^{-1}$ pixel $^{-1}$ both interior and (to a lesser degree) exterior to portions of the debris ring itself. This is the cause of the low-level ringlike artifact in the Figure 9(C) (2015-2001) difference image at the same location of the ring as in panels (A) and (B). Earlier attempts to empirically mitigate this recognized undersubtraction (Schneider $2001^{11}$ ) were partially successful (Schneider et al. 2009, their Figure 8), but use of HR 4735 as a

\footnotetext{
11 Also see http://nicmosis.as.arizona.edu:8000/AAS_2001_JUNE_ HR4796A.ppt.
} 


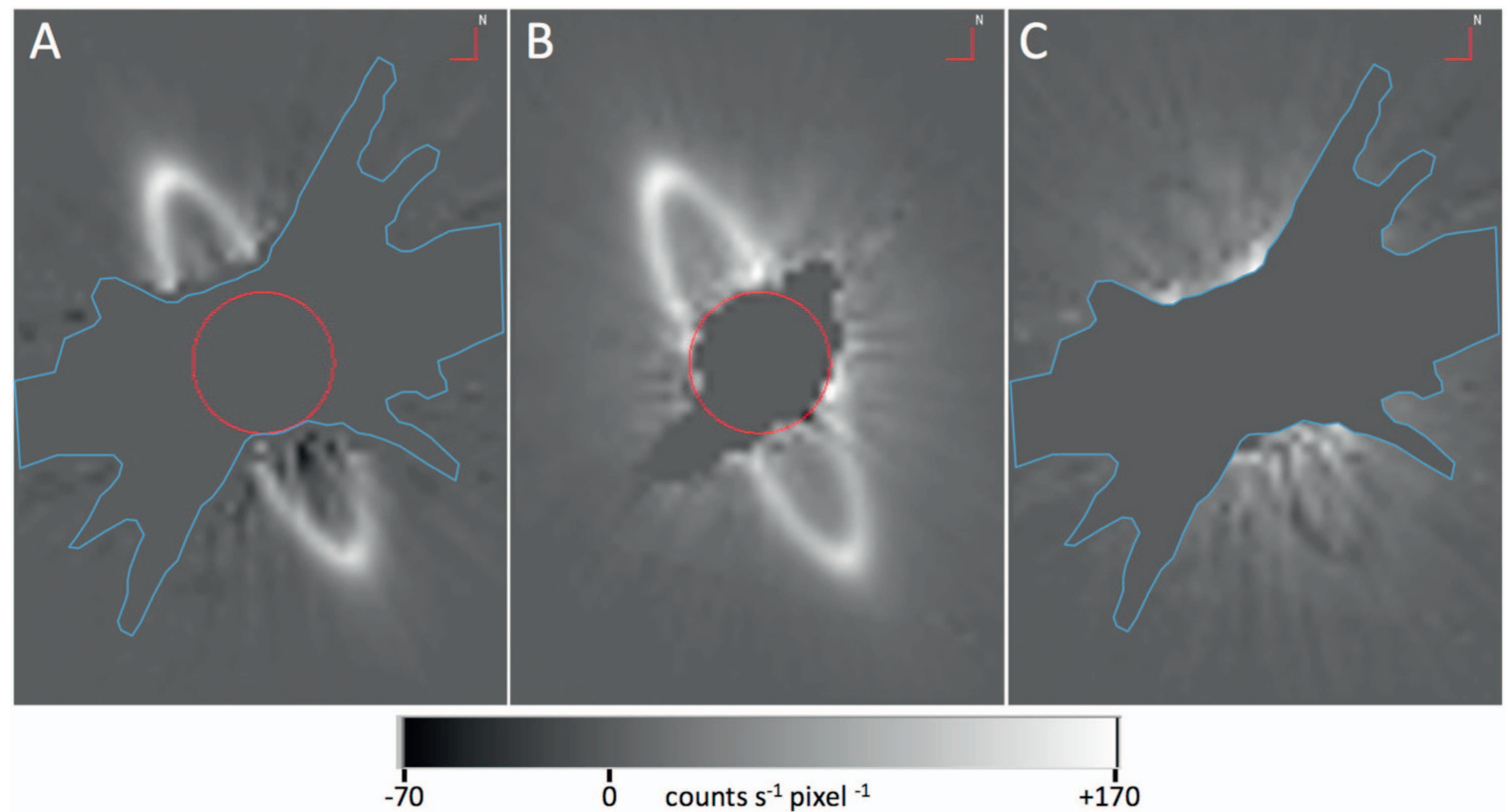

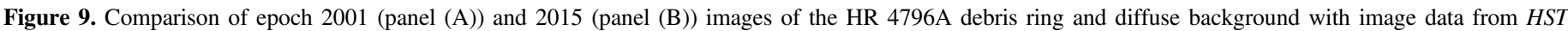

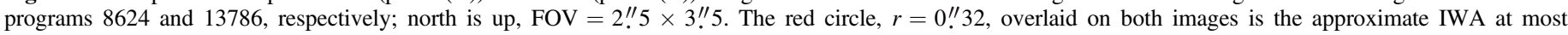

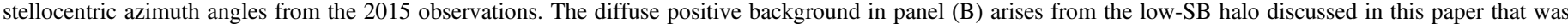

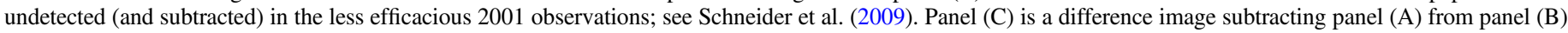

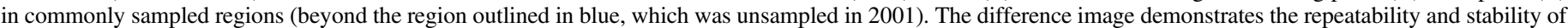
the debris ring imaging approximately at or below the level of the differences in the 2015-2001 PSF-subtraction residuals.

contemporaneous PSF template in GO 13786 fully obviated this issue in the epoch 2015 imaging.

Despite the local bias in the GO 8624 image background, the efficacy of its ring photometry is evidenced in the epoch 2015 minus 2001 difference image (Figure $9(\mathrm{C})$ ). In that image, the ring all but disappears among the difference in PSF-subtraction residuals in commonly sampled areas, and the inter-epochal brightness at both ansae before differencing is statistically identical. Note that in GO 13786, along with BAR5 coronagraphy, the observations and data also included much deeper and independent WedgeA-1.0 imaging. This permitted a verification (reproducibility and continuity) of brightness at the level of the lower-SB dust detected in the exo-ring region of overlap between $\approx 1$ !" 2 and 2 !" 6 in the 2015 epoch data (Figure 7, panels (A) and (B)).

\section{Principal Observational Results}

\subsection{Systemic Extent}

Figure 8 shows the previously unimaged full extent and asymmetrically complex morphology of the HR 4796A debris system. Beyond the earlier imaged region of the high-SB, $r=1$ ". 05 debris ring itself, we now map in visible light the starlight-scattering material in the exo-ring environment over $\sim 5$ dex in SB. To the northeast of the star, material is seen extending in a smoothly contiguous fanlike radial structure in and "above" (to the northeast) the extension of the debris ring major axis to a stellocentric distance $\geqslant 12^{\prime \prime}$ from and above this brighter of the two ring ansae. This material is detectable to a $1 \sigma$ pixel $^{-1}$ background-limiting noise level of 0.042 $( \pm \sim 15 \%)$ counts s ${ }^{-1}$ pixel $^{-1} \quad\left(=24.0 V_{\text {mag }} \operatorname{arcsec}^{-2}\right.$ for spectrally neutral dust) assessed at $12^{\prime \prime}<r<15^{\prime \prime}$ (in the northwest and southeast corners of the Figure 8 field). To the southwest of the star, exo-ring material in the diametrically opposed half of the debris system (in roughly the direction toward the location of the PSF-subtracted M-star companion) is seen with radially declining $\mathrm{SB}$ along the extension of the ring major axis to an angular distance of $\approx 4$ !" 5 , but it then "bends" or "kinks" toward the south to a stellocentric angular distance of $\approx 5 ! \prime 7$ at celestial P.A. $\approx 190^{\circ}$. That is, the faint material on the northeast side of the exo-ring debris structure extends to more than twice the angular distance of the dust at the southwest side periphery.

\subsection{Exo-ring Major-axis SB Profile Asymmetry}

A major-axis radial SB profile, $\pm 16^{\prime \prime}$ from the debris ring center, is presented in Figure 10. The profile is measured in a 1 pixel wide strip along a symmetrical extension of the debris ring major axis (green line in the corresponding image above the profile in Figure 10). The photocentric brightness peaks near the ring ansa (Figure $7(\mathrm{C})$ ) are displaced (due to directionally preferential forward scattering) with respect to a stellocentric position by 0.643 pixels ( $=32.7$ mas) perpendicular to the ring minor axis toward the southeast. The radial brightness gradient of the exo-ring material, where seen on the bidirectional extension of the ring major axis, is less steep on the more greatly extended northeast side than on the southwest side. Along the major axis to the southwest of the star, the debris dust SB is closely approximated by a single power-law fit with $\mathrm{SB} \sim r^{-5.1}$ (goodness of fit: $R=0.980$ ) where dust-scattered starlight is robustly detected at $r<4$ ".5. To the northeast of the star, the corresponding profile may be closely fit by three contiguous power laws of lesser declining steepness from (a) the ansal peak at $r=1$ !"04 to $r=1$ !" 6 steeply declining as $r^{-7.8}$ (goodness of fit: $R=0.978$ ), (b) 1 !' $6<r<4$." 5 with $\mathrm{SB} \sim r^{-3.6}(R=0.977)$, and (c) 4". $5<r<12^{\prime \prime}$ with $\mathrm{SB} \sim r^{-2.7}(R=0.967)$. Region (c) is beyond the angular extent of the exo-ring material on the southwest major axis. The photometric uncertainties (internal 


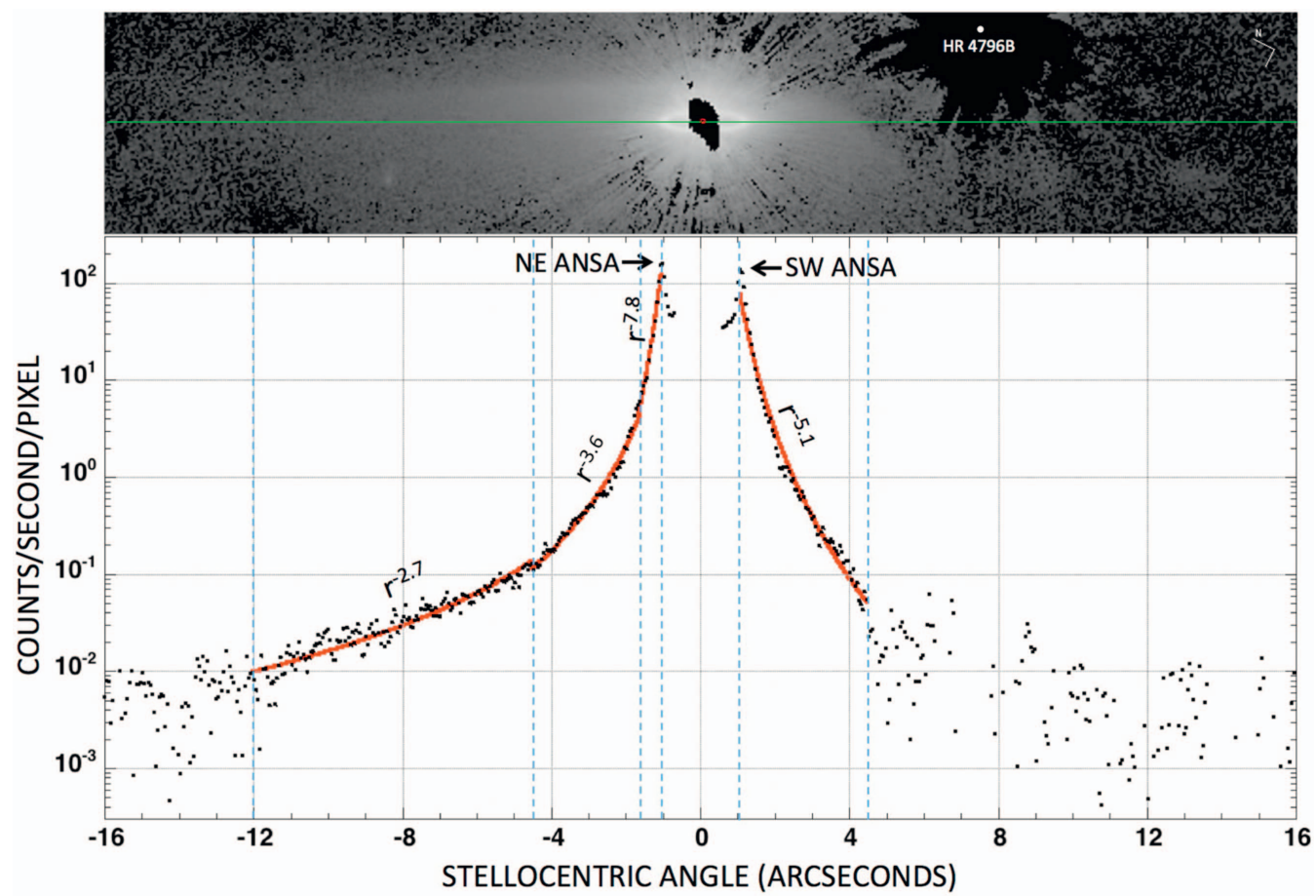

Figure 10. Plot of a 1 pixel $\left(0{ }^{\prime \prime}\right.$ 05077) wide radial SB profile of the HR 4796A debris structure with corresponding image and power-law fits. Along the extension of the debris ring major axis (green line), starlight-scattering debris is robustly detected to $r \approx 12^{\prime \prime}$ on the northeast side of the star, but on the southwest side it is detected only to $\approx 4$ !" 5 with an abrupt truncation or outer-edge boundary as the periphery of the debris structure "bends" to the south.

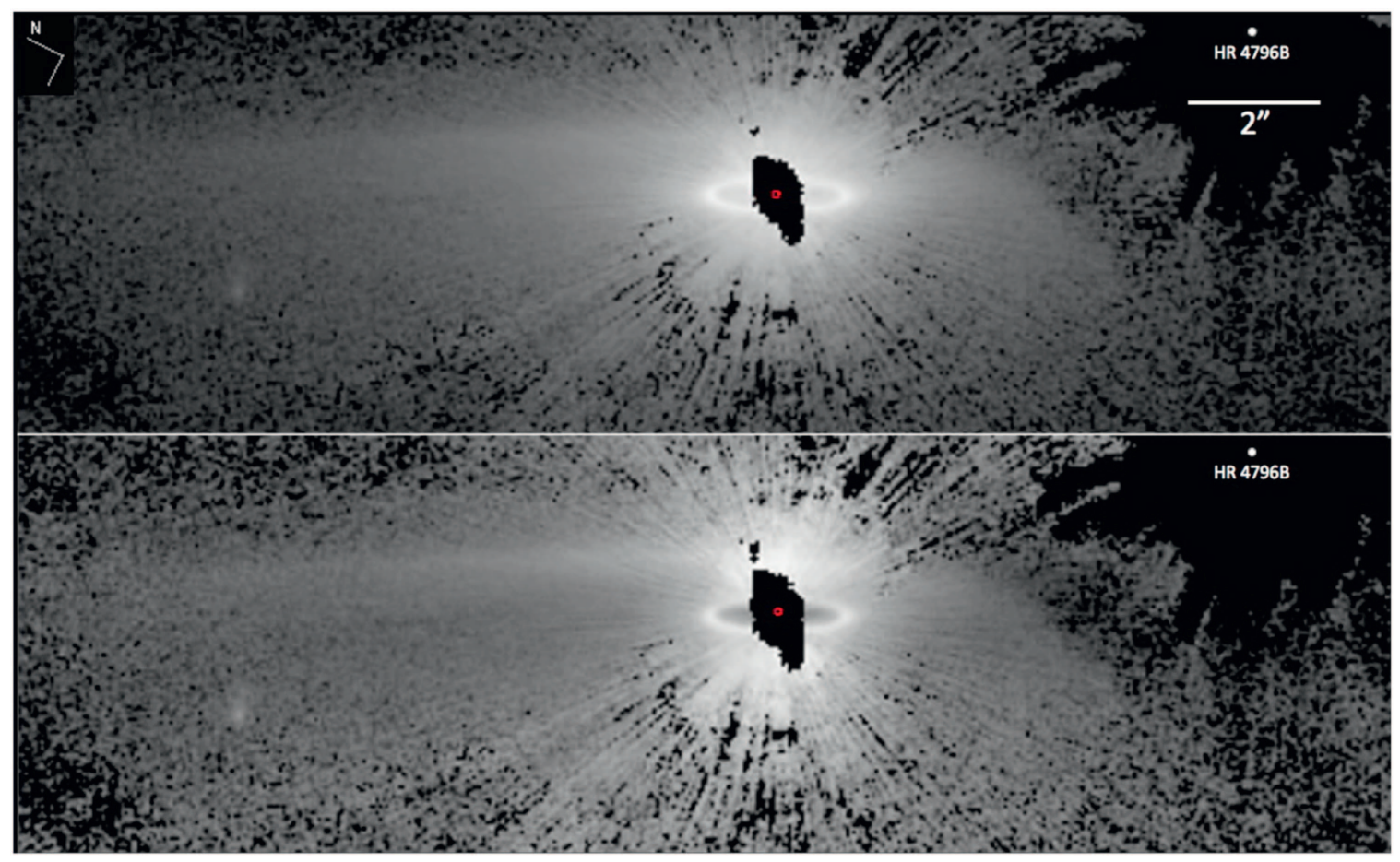

Figure 11. HR 4796A debris structure and its morphological "leading edge." Top: SB image in scattered light enclosed within a 6". $2 \times 20^{\prime \prime}$ field extraction. Same data (and display stretch) as in Figure 8 (left) but rotated to put the debris ring major axis (P.A. $=26^{\circ} 37$, rederived from these data; see Table 4) on the image horizontal. Bottom: with compensation for the $r^{-2}$ diminution of the starlight illuminating the scattering material in the plane of the disk, normalized to the SB peak at the brighter (northeast) ansa. This image suggests regions of relatively lower surface density of scattering particles both interior to the $r=1$ !' 05 debris ring and between it and the exterior leading edge of scattering material on the south side of the debris system; see annotated Figure 13.

measurement errors) of the individual points are closely approximated by the local dispersion around these sectional radial power-law fits: a few percent at $r<4$ ". 5 on both sides of the star and increasing to $\sim 15 \%$ at 12 " on the northeast side of the star at the outer detectable periphery of the debris system, as enumerated in Section 6.1. 


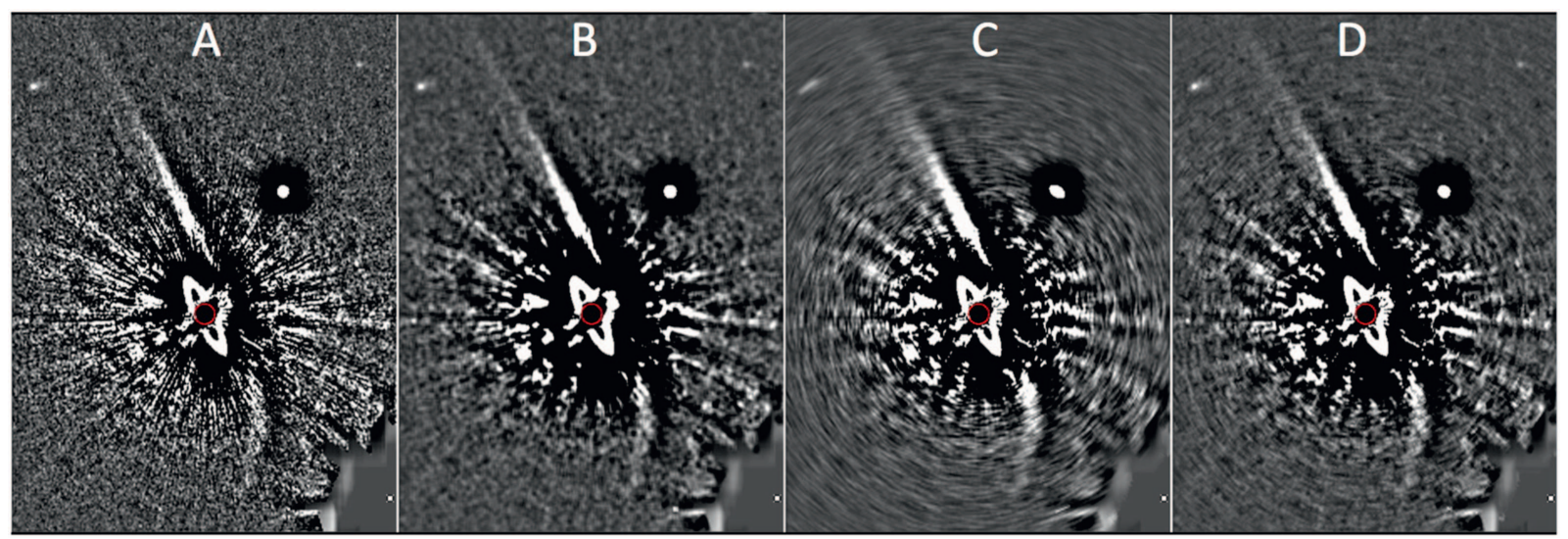

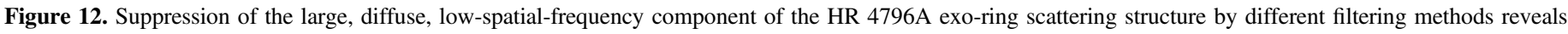

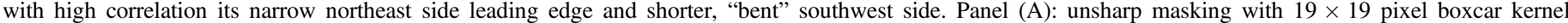

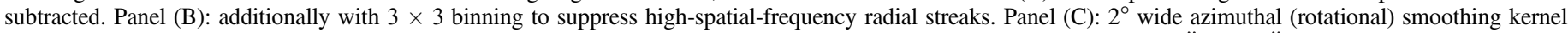
subtracted. Panel (D): panels (A)-(C) all applied with equal weight. All panels are north up, east to the left, with FOV $11^{\prime \prime} 7 \times 15^{\prime \prime} 5$.

\subsection{Morphology}

The material exterior to the southwest side of the debris ring, unlike on the northeast side, apparently "bends" to the south, where it appears terminated or truncated on the major axis at a stellocentric distance of $\approx 4$ ". 5 . Together, the two asymmetric halves of the exo-ring debris structure give the system a distinctive boomerang-like morphology with a visual appearance suggesting a brightening along the "leading edge" (centered on the apex of the boomerang) to the northwest side of the host star. See Figure 11. For purposes of morphological interpretation, the dust structure is seen with enhanced clarity by compensating for the $r^{-2}$ falloff in the stellar illumination of the scattering material in the disk plane as seen in sky-plane projection. We do so, comparatively, as shown in Figure 11 (bottom panel). This is a first-order proxy ${ }^{12}$ to a radial surface density image. In this simple image transformation, we have assumed that the exo-ring dust is coplanar (or nearly so) with the debris ring. ${ }^{13}$

An examination of Figure 11 (both panels) reveals a contiguous shallow arc (seen in projection with the system inclination) of scattering material with a relatively abrupt brightness gradient across its edge, azimuthally subtending a stellocentric angle $\approx 195^{\circ}$ roughly centered at celestial P.A. $=287^{\circ}$. Figure 11 (bottom panel) suggests that this "edge" is a radial region of enhanced surface density relative to a more depleted (darker) region closer to the star between the bright debris ring and this outer structure edge.

Separately, as an additional check on the identification of this feature, various forms of spatial filtering were applied to the SB image. These digital filters eliminated the diffuse lower spatial frequency exo-ring scattering structure, though are nonconservative in photometry. Together, these filtered images provide a robust demonstration in confirming the location and

\footnotetext{
12 A higher-fidelity transformation from a SB to surface density image would also compensate for azimuthally anisotropic scattering. While such a scattering function of an assumed form (e.g., Henyey \& Greenstein 1941) can be empirically determined for the debris ring itself, unconstrained extrapolation of an inner ring asymmetry parameter to the outer periphery of the much-larger exo-ring debris structure is not (yet) well founded.

13 We adopt an inclination for the debris ring of $75^{\circ} .9$ that we derived from the 2015 epoch image shown in Figure 7(B). This is in statistically identical agreement with the prior determination by Schneider et al. (2009) from the 2001 epoch image (Figure 7, and see Table 4).
}

morphology of this leading-edge debris system feature on both the northeast and southwest sides of the star. See Figure 12.

\subsection{Photometry and Spatially Resolved SB}

Prior high-contrast scattered-light imaging of HR 4796A, either space-based in total light or ground-based with polarized intensity measurements, has well revealed its high-SB, spatially compact debris ring; see Figure 13 and its captioned citations. However, such AO images (panels (B)-(G)), also with angularly small high-contrast FOVs, reveal circumstellar materials only within the debris ring itself. These prior observations, however, were insensitive to then-only-posited lower-SB exo-ring material that is now is revealed with significance to a much greater angular extent with STIS 6R/ PSFTSC. In Figure 13, we provide a two-dimensional fluxdensity-calibrated SB map of the debris system enclosing to a much greater extent the a priori known high-SB debris ring (in panels (A)-(G), reproduced at $1.7 \times$ spatial scale). After starlight suppression, starlight-scattering material is detected over a brightness range of $\approx 13.5 \mathrm{mag} \operatorname{arcsec}^{-2}$ declining from the peak brightness of the debris ring to the debris system periphery.

In the inner part of the system, our new BAR5 imaging confirms: (a) the visible southwest-to-northeast-side brightness asymmetry, (b) characteristic FWHM of the ring at the ansae (unaffected by sky-plane projection), and (c) steep endo-ring inward decline in the disk SB along its major axis, as previously reported by Schneider et al. (2009). As informed by our new images, (d) the fainter (southwest) side of the debris ring corresponds to the truncated side of the larger debris system, (e) the measured FWHM of the ring at the northeast and southwest ansae are 224 and 244 mas, respectively, ${ }^{14}$ and (f) the best power-law fits to the inner-edge $(0$ "' $85<r<1$ !" 03$)$ ring slopes from a radial SB profile along the disk major axis (Figure 14) are $\mathrm{SB} \sim r^{-6.5}(R=0.994)$ and $\mathrm{SB} \sim r^{-6.0}$ $(R=0.992)$ across the northeast and southwest ansae, respectively. We infer the latter as arising from a dearth of scattering particles that are indicative of a centrally cleared disk.

\footnotetext{
${ }^{14}$ By subtracting in quadrature the FWHM of an STIS 50CCD resel, the intrinsic widths are 210 and 234 mas (=15.3 and 17.0 au at $72.8 \mathrm{pc})$.
} 


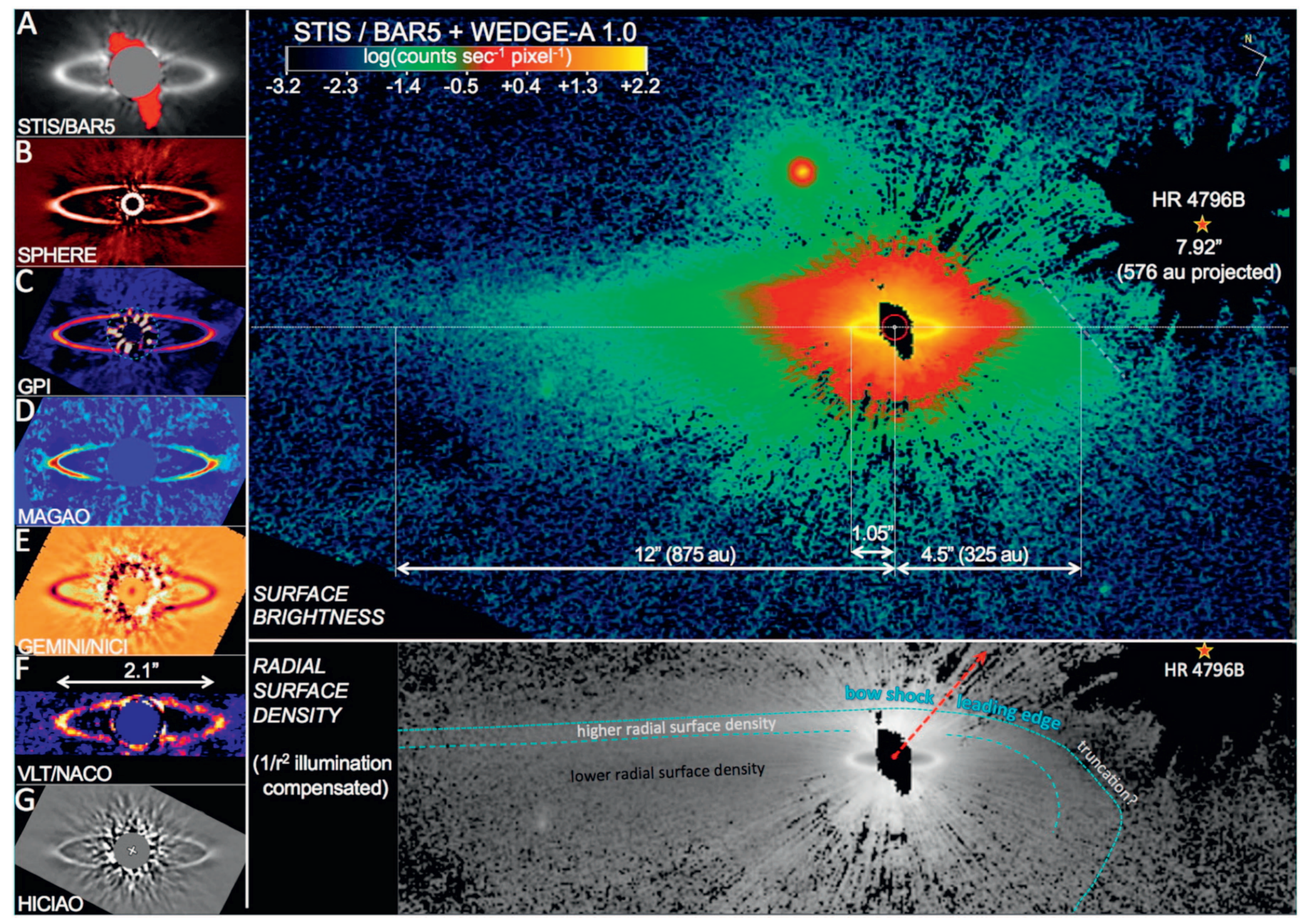

Figure 13. As with STIS/BAR5 (panel (A)), ground-based images of the HD 4796A disk with various postprocessing methods reveal its very bright $\left(f_{\text {disk }} / f_{\text {star }} \approx 0.1 \%\right)$ debris ring: (B) ESO (2014), (C) Perrin et al. (2015), (D) Rodigas et al. (2015), (E) Wahhaj et al. (2014), (F) Lagrange et al. (2012), (G) Thalmann et al. (2011), but with an estimated up to $\sim 75 \%$ flux loss from contrast-enhancing PSF-reduction (e.g., Perrin et al. 2015). The same ring imaged with STIS 6R/PSFTSC (top left) was extracted from a much larger stellocentric field (top right) with spatial resolution 72 mas resel ${ }^{-1}$ in visible light to the sensitivity limits of STIS 6 R/PSFTSC with $\approx 9.3 \mathrm{ks}$ of total integration time. The debris system, exhibiting a leading-edge bow shock and a morphological one-side truncation in the direction toward its close-proximity companion, is photometrically mapped in the top right panel and shown with these key features annotated in $r^{-2}$ scaled enhanced form in the bottom right panel.

\subsection{Total Flux Density}

As a close lower limit, the $0.58 \mu \mathrm{m}$ flux density of the debris system, fully enclosed by a $30^{\prime \prime} \times 10^{\prime \prime}$ photometric aperture centered on HR 4796A with a long dimension parallel to the disk major axis measured from the AQ image, is $\approx 27.6 \mathrm{mJy} .{ }^{15}$ This includes flux due to the debris ring and from the larger enclosing exo-ring scattering structure. This measurement, however, excludes the small unsampled region asymmetrically flanking the ring minor axis close to the star (red regions in Figure 15, panels (A) and (C)) and interior everywhere at $r<0$ ". 32 (circular gray regions). To estimate most of the unmeasured flux in these regions, we employ a simple twocomponent model. For the debris ring itself, we use a scatteredlight model as described by Sch16 best fit to the unobscured portion of the ring (Figure 15, panel (B), with principal geometrical parameters given in Table 4). For the scattered light from the diffuse dust due to the exo-ring structure, after

\footnotetext{
15 In making this measurement, we subtract the small amount of additional light from the background star at $\approx 4$ ! 5 to the northwest of HR 4796A (see Figure 8 ) by direct-image PSF subtraction using a Tiny Tim model PSF.
}

digitally masking light from the debris ring (panel (D)), we perform a two-dimensional interpolation of nearest-neighbor pixels that flank the regions in red and smooth by a 3 pixel wide Gaussian kernel (panel (E)). These two (panels (B) and (E)) components combined are shown implanted in the unsampled data region in panel (F). From this, we more closely estimate the total flux density of the debris system as $\approx 32.3 \mathrm{mJy}$. In this estimation, only the flux from the central $\approx 0$ ". $36 \times 0$ ". 68 near-rectangular endo-ring region (black in panel (F)), with no firm basis for estimation, remains unaccounted and likely only further contributes a very small fraction to the total debris system $0.58 \mu \mathrm{m}$ flux density.

The fraction of circumazimuthal flux from the system (excluding all background sources) beyond the prior posited $r \approx 1$ ". 5 outer edge of the debris ring to a stellocentric distance of $\approx 12^{\prime \prime}$ contributes only about $\approx 12 \%$ of the total system optical brightness. Much of this flux is out of the plane of the disk. Given the system inclination to the line of sight, however, the rough red-to-green and green-to-blue isophote axial ratios in Figure 13 suggest near coplanarity of the exo-ring material and the material in the debris ring but with some vertical 


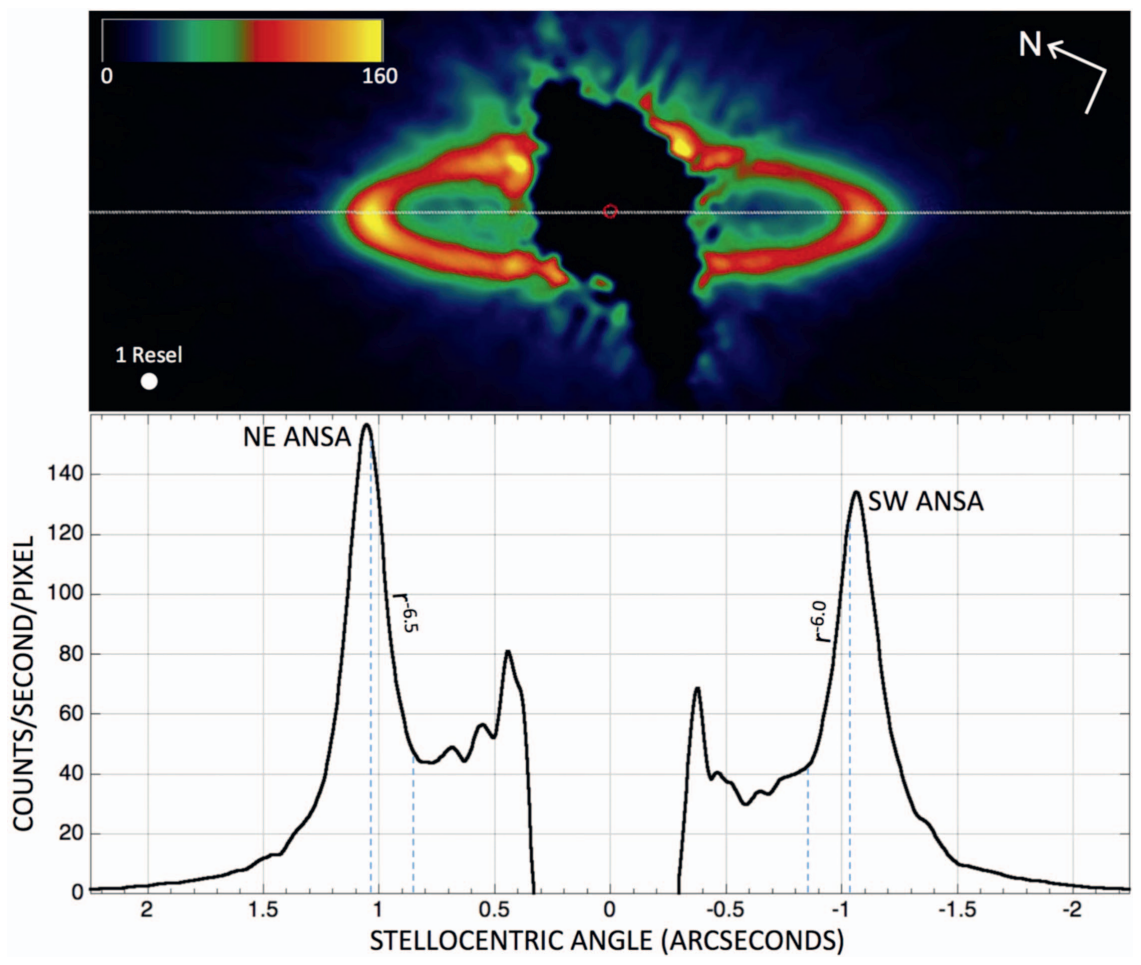

Figure 14. Top: SB image of the HR 4796A debris ring with a linear display stretch maximum corresponding to the SB peak at the northeast ansa. Bottom: corresponding 1 pixel ( $\left.0 .^{\prime \prime} 05077\right)$ wide radial SB profile along the disk major axis (white line in the top panel). The regions containing the debris ring inner-edge slopes, fit by radial power laws, are indicated by the blue dashed lines. (Residual image artifacts close to the unsampled central region, in convolution with PSF broadening due to the finite size of the STIS beam, are responsible for the nonastrophysical upturn in flux at $r<\sim 0$." 5 .)
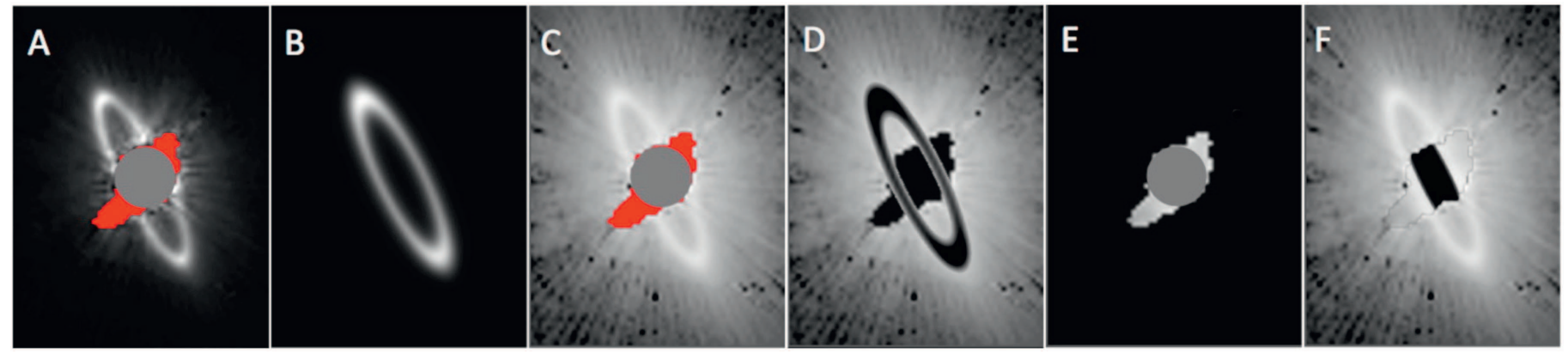

Figure 15. (A): HR 4796A debris ring image (linear stretch $0-160$ counts $\mathrm{s}^{-1}$ pixel $^{-1}$ ) with regions unsampled due to the imposition of BAR5 flanking the minor axis in red. (B): best-fit model of the debris ring following Sch16. (C): same as panel (A) but $\log _{10}$ stretched from [-1.5] to [+2.5] counts s$^{-1}$ pixel $^{-1}$ to simultaneously show lower-SB dust-scattered starlight in the exo- (and endo-) ring regions within a few arcsec of the star. (D): using the best-fit ring model morphology as a digital mask to obscure the ring itself, the unsampled SB distribution is estimated (panel (E)) - see main text. (F): estimation of the unsampled light from B $+\mathrm{E}$ implanted (superimposed) on the observed disk image. All panels are north up, east to the left, with FOV $3{ }^{\prime \prime} 0 \times 3$ !' 9 .

broadening by some mechanism(s) that may also contribute to the debris system asymmetries.

\subsection{Geometrical Parameters}

The high level of astrophotometric instrumental stability of STIS coronagraphic imaging was recently demonstrated over a temporal baseline of $18 \mathrm{yr}$ with a revisit to the $\beta$ Pictoris system (Heap et al. 2000; Apai et al. 2015). Using STIS two-roll PSFTSC, Schneider et al. (2009) derived the key characterizing parameters of the HR 4796A debris ring from epoch 2001 50CCD spectral-band imaging (see Figure 9(A)). While the focus of our new observations in 2015 was on HR 4796A's exo-ring environment, we advantageously obtained BAR5 6R/ PSFTSC dither-combined imaging (see Figure 9(B)) to compare over time the previously determined geometrical parameters of the debris ring in the same spectral band and (except for the coronagraphic mask used) the same instrumental configuration. Comparative measurements of the high$\mathrm{S} / \mathrm{N}$ debris ring images from 2001 (as published) and 2015 were made in the manner described by Schneider et al. 2009 . Despite differences in detail in the observation and reduction methodologies (see Section 4), no changes of statistical significance were found; see Table 4.

\section{Discussion}

HR 4796A now joins a small but growing number of exoplanetary debris systems with ringlike architectures that also possess very large now-imaged exo-ring structures 


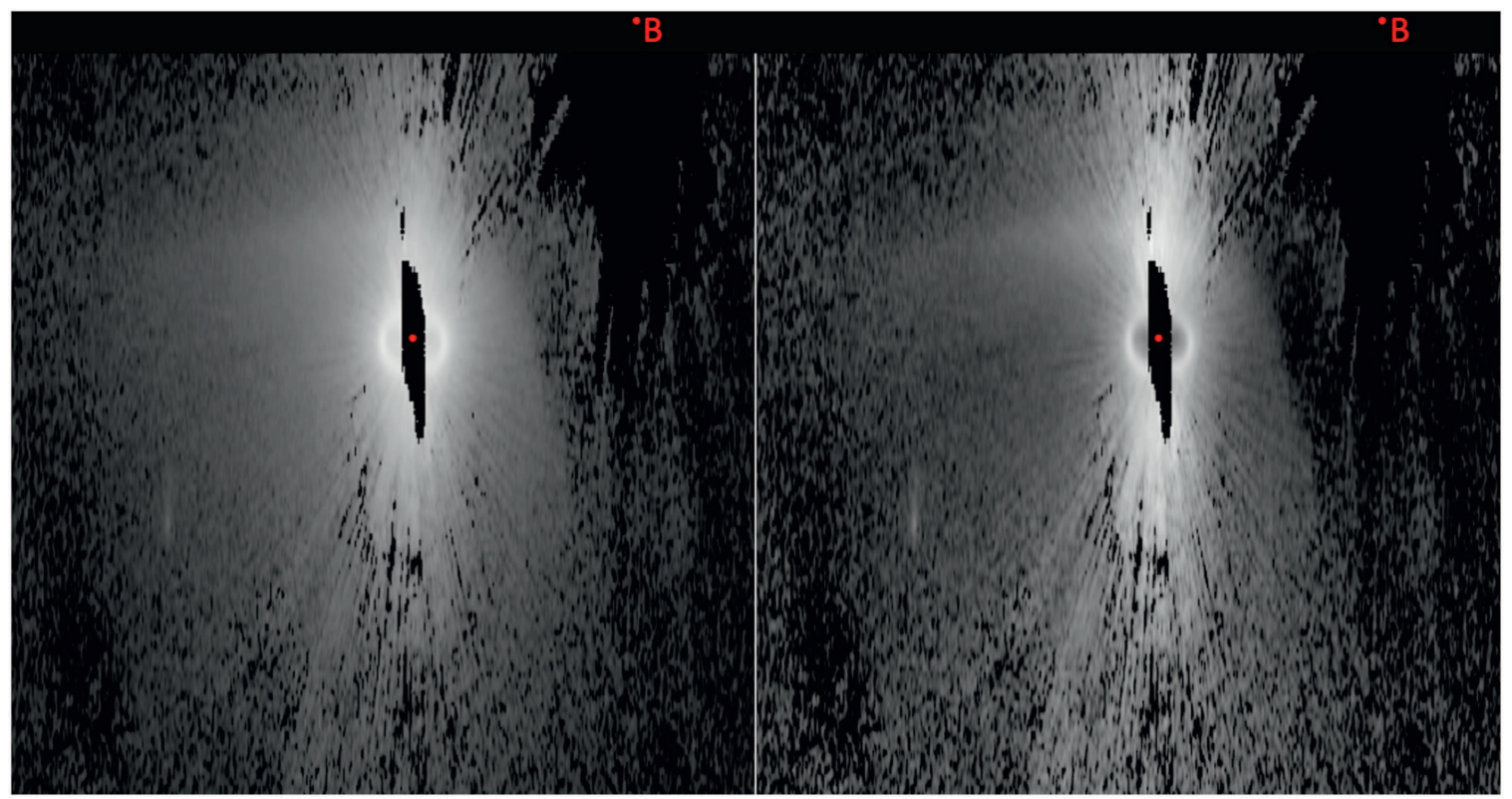

Figure 16. Face-on geometrically projected images of (left) the HR 4796A surface brightness and (right) an approximation to its radial surface density (1/ $r^{2}$ scaled in the plane of the debris ring assuming coplanarity of scattering materials). In detail, these images are rotated by $63^{\circ} .6$ from north up to place the deprojected debris ring major axis on the image horizontal and geometrically projected assuming a global system inclination of $75^{\circ} 9$ (see Table 4 ). The FOV before deprojection (in the skyplane geometry oriented with the debris ring major axis on the image horizontal) was 25 !" $3 \times 6$ !" 3 ; in deprojection, the physical field size is 1850 au on a side. A "B" marks the deprojected location along the line of sight of HR 4796B.

Table 4

Geometrical Parameters of the HR 4796A Debris Ring: Epochs 2001 and 2015

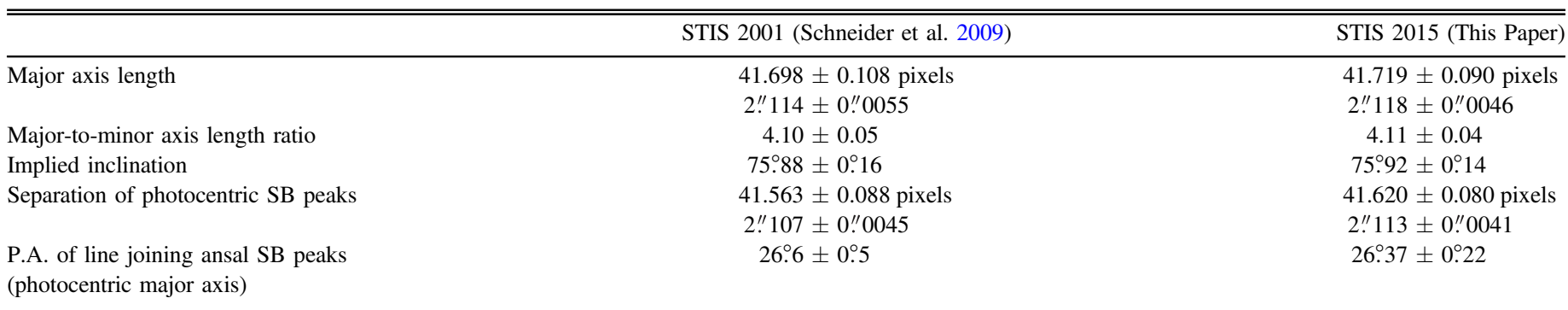

comprised of small (micron-size) starlight-scattering particles seen in visible light (e.g., Sch14; Sch16; Konishi et al. 2016). Such structures, though technically challenging (if not problematic) to observe with current ground-based high-contrast imaging instrumentation and techniques, may not be uncommon, in particular for younger systems, though only a few have been observed (with $H S T$ visible-light coronagraphy) to date.

The morphology of the HR 4796A debris system is both highly complex and biaxially asymmetric beyond its bright debris ring (see Figure 13, right panels). On the northeast side of HR 4796A, starlight-scattering particles are detected to a stellocentric distance of $\geqslant 12^{\prime \prime}$ (875 au; 12 times larger than the debris ring radius itself) along the extension of the debris ring major axis. The ridge of brightest isophotes, however, does not extend along the debris ring major axis. Rather, an enhancement in both the radial SB and density $\left(1 / r^{2}\right.$ scaled) profiles are seen to the northwest side of the debris ring's sky-planeprojected major axis, morphologically resembling a leadingedge bow shock. On the opposite (southwest) side of the host star, the exo-ring halo appears truncated at a midplane radial distance of $\approx 4$ ". 5 (325 au, projected) in the direction toward HR 4796B (see dashed line overlay in Figure 13, top right panel).
To better visualize the intrinsic morphology and full circumazimuthal extent of the exo-ring structure, in Figure 16 we deprojected the system to a face-on viewing geometry. In doing so, to first order, we simply assume that the entire structure is coplanar with the bright debris ring, though some materials may indeed be located above or below the plane of the ring. This approximation nonetheless helps to visualize: (1) the "boomerang"-like morphology of the forward edge of the bow shock, (2) the rather abrupt "kink" in its periphery to the southwest of its apex in the direction toward HR 4796B, and (3) the truncation of the southwest side/extent of the exoring region.

Deep, high-contrast coronagraphic imaging with PSF template subtraction for further augmentation of starlight suppression, as uniquely performed with the HST, has previously succeeded in revealing very low SB diffuse, and spatially extended dust structures over a large range of stellocentric angles associated with several $\sim 10-100 \mathrm{Myr}$ debris disk-hosting stars, including, e.g., HD 181327, HD 61005, HD 32297, and HD 15115 (Sch14). HR 4796A, discussed herein, was technically more challenging because of the additional presence of its close angular proximity M-star companion, HR 4796B, but was overcome to requisite contrast 


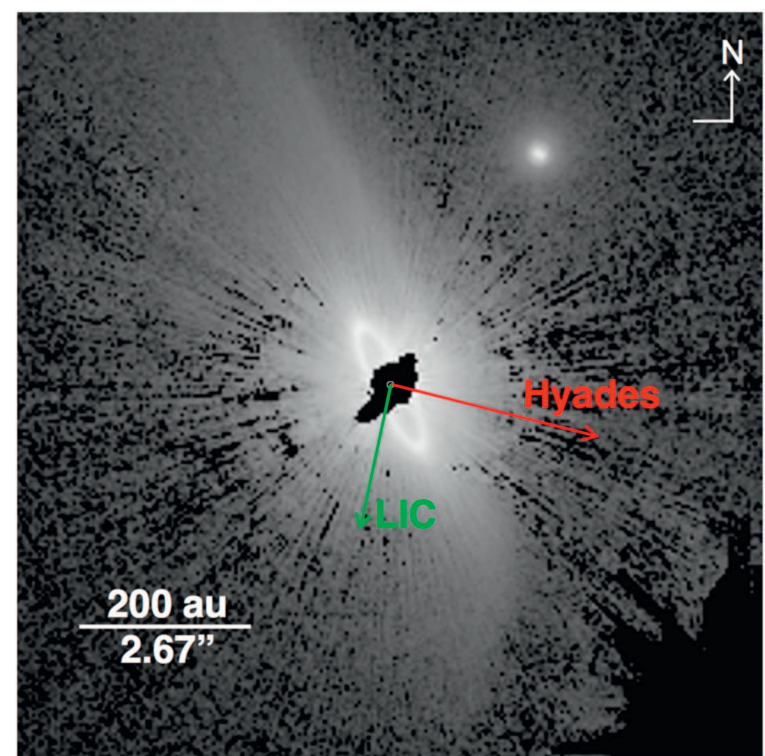

Figure 17. Relative motion of the HR 4796A exoplanetary debris system through the LIC and Hyades Cloud over $100 \mathrm{yr}$ (length of arrows).

levels through simultaneous PSF subtractions described in Section 4 to fully reveal its exo-ring structure.

The presence of M-star companion(s) at similar distance(s) to A-star disk hosts is not unique to HR 4796A. HD 141569A (Weinberger et al. 2000; Clampin et al. 2003; Konishi et al. 2016), an $~ 5$ Myr Herbig Ae star with a mature transitional-stage disk, possesses two close-proximity M-star companions that have been posited as causal for an arc-like structure extruding from the outer of its two "nested" bright rings of starlight-scattering material through tidal interaction. No such structure is seen in the HR 4796A debris system. It does, however, possess a "leading edge" higher SB/density arc-like structure suggestive, instead, of a bow shock. This, in some regards, is morphologically most similar to HD 61005 (Hines et al. 2007; Schneider et al. 2014) and $\delta$ Vel (Gáspár et al. 2008). A similar causal mechanism for such a structure, as suggested for several other debris disks (noted above plus HD 202917 (30 Myr) and HD 15745 (30-200 Myr); Sch16) posited as interacting with local ISM clouds may also be in play here. In Figure 17, we show the relative motion of the HR 4796A system with respect to the Local Interstellar Cloud (LIC) and the Hyades Cloud, based on Redfield \& Linsky (2008), over 100 yr. The LIC is less likely plausible, but the motion with respect to the Hyades Cloud $\left(\Delta \mathrm{V}[\right.$ tangential, radial $\left.]=[+12.0,-18.1] \mathrm{km} \mathrm{s}^{-1}\right)$ is a good match for producing the leading-edge bow shock seen.

The large exo-ring halo surrounding HD 181327 may result from radiation pressure blowout of small grains recently released from a massive collision in the interior birth ring (Stark et al. 2014), whereas the highly asymmetric exo-ring structures of HD 61005 (Hines et al. 2007; Maness et al. 2009) and HD 32297 (Debes et al. 2009) may be caused by ram-pressure interaction with the ISM, with telltale bow-shock-like features on their inferred leading edges and blowback "fans" on their trailing sides. The origin of the bifurcated exo-ring structure one side only of HD 15115 (Sch14) is not yet clear.

The presence of a stellar companion may also influence and/or disrupt the spatial distribution of small grains in the HR 4796A exo-ring halo. This had been suggested for the HD 141569A ( 5 Myr Herbig Ae star) disk by Clampin et al. (2003) through tidal interactions with its two M-star companions (Weinberger et al. 2000) and posited for HR 4796A with its similarly distant M-star companion, though its exo-ring structure had previously been unobserved for (then) lack of detection sensitivity. The morphology of the HR 4796A debris system suggests that the presence of its M-star companion, at a projected distance of $\sim 580 \mathrm{au}$, may affect (truncate, redistribute, or expel) the small grains at large distances on the southwest side of the disk and speculatively may also be responsible for the "kink" in the debris system morphology on that side of the exo-ring structure.

Separately, the apparent outer-edge truncation of the $r=1$ !"05 (77.1 au) debris ring itself, seen in earlier scatteredlight imaging (e.g., Schneider et al. 1999, 2009 and as shown in Figure 13; also observed with ground-based AO imagers), previously led to both suggestions for and constraints (e.g., high orbital eccentricity) against its causality (at least in part) due solely to the system's M-star companion (e.g., Augereau et al. 1999; Thebault et al. 2010; Lagrange et al. 2012). The new STIS observations, with much greater sensitivity to lowSB small particles beyond the posited outer-truncation radius, clearly show a low-SB continuum of small particles seen to a very large stellocentric distance far beyond the debris ring itself on one side of the disk but truncated on the opposite side.

At least three potentially plausible mechanisms are postulated for the apparent southwest outer-edge truncation of the exo-ring material due to HR 4796B: (1) dynamical interactions (spoken to above), (2) radiation pressure, and (3) corpuscular winds. Arbitrating between these possibilities is the subject of future work beyond the scope of this paper, but we generally comment as follows. (1) Assuming a mass of $2.18 M_{\odot}$ for $\mathrm{HR} 4796 \mathrm{~A}$ (Gerbaldi et al. 1999), the minimum orbital timescale for HR 4796B $\left(0.3 M_{\odot}\right.$ Huélamo et al. 2004) with a projected separation of $576 \mathrm{au}$ is approximately $9000 \mathrm{yr}$. Parent (and other) particles in the bright debris ring orbit with periods of $\approx 450 \mathrm{yr}$. Those at the southwest leading-edge truncation radius of $325 \mathrm{au}$, if bound, orbit with a period of $\approx 4000 \mathrm{yr}$, and dynamical truncation may be possible over many periastron passages with a higheccentricity companion orbit (e.g., Thebault et al. 2010). (2) The distance to the southwest truncation edge (along the debris ring midplane) is 325 au from the central A0V $\left(23 L_{\odot}\right)$ star and not largely different (projected minimum $275 \mathrm{au}$ ) from the M2.5V (0.3 $L_{\odot}$; Huélamo et al. 2004) companion. Thus, the radiation pressure from the $\sim 76 \times$ more luminous primary star would greatly dominate over the companion with blowout particles where the truncation is seen. (3) Chromospheric activity that can drive corpuscular winds is not uncommon for early/ young M stars such as HR 4796B. This is the case, e.g., for the similarly young $(\approx 12 \mathrm{Myr})$ debris disk host star AU Mic (spectral type M1V), for which the radial component of seemingly super-Keplerian motions of debris structures have been seen in its edge-on disk (Boccaletti et al. 2015). Though the level of chromospheric activity in HR 4796B itself is not well established, it is X-ray active with a ROSAT/HRI X-ray flux of $2.8 \times 10^{-13} \mathrm{erg} \mathrm{cm}^{-2} \mathrm{~s}^{-1}$ (Huélamo et al. 2000).

\section{Summary and Concluding Remarks}

With the HST/STIS six-roll PSFTSC and contemporaneous use of the STIS WedgeA, WedgeB, and BAR5 occulters, we have holistically explored the morphology, structure, and extent of the HR 4796A exoplanetary debris system with deep, visiblelight imaging and photometry. These observations sensitively probe a very large stellocentric angle range with spatially 
resolved, diffraction-limited imaging that maps the debris system far into its exo-ring environment. Specifically, from these images we find the following.

(1) The optically bright, $r=1$ !'05 HR 4796A exoplanetary debris ring is revealed for the first time to be embedded within a much larger, morphologically complex, and biaxially asymmetric exo-ring scattering structure seen at visible wavelengths sensitive to micron-size particles with HST/STIS multi-roll PSF template-subtracted coronagraphy also addressing background-light rejection from its $\mathrm{M} 2.5 \mathrm{~V}$ close-proximity companion.

(2) Starlight scattered by the exo-ring particles is detected to a distance of at least $12^{\prime \prime}(875 \mathrm{au})$ to the northeast of the host star to a $1 \sigma$ background-limiting noise level of $\approx 24.0 V_{\text {mag }} \operatorname{arcsec}^{-2}$.

(3) The exo-ring debris system is morphologically truncated to $r \approx 4$ ". 5 (325 au) on the southwest extension of the debris ring major axis, the side of the star in approximately the direction toward the projected location of its M2.5V companion HR 4796B.

(4) Contemporaneous imaging with HST's smallest coronagraphic BAR5 occulter confirms an endo-ring clearing (particle depletion) at visible wavelengths to an instrumentally limited effective IWA of $r=0$ "! 32 (23 au).

(5) The exo-ring material, beyond the previously considered bright ring outer "edge," in the range 1 ". $5<r<12$ ", contributes approximately $12 \%$ to the total light of the debris system at visible wavelengths.

(6) To the southwest of the star, from the peak SB of the ring outward to the point of truncation along the extension of the debris ring major axis, the SB is well represented by a single power law with $\mathrm{SB} \sim r^{-5.1}$. On the opposite (northeast) side of the star, a three-component power law well fits the observed SB profile with the asymmetrical (untruncated) outer region $\mathrm{SB}(r \geqslant 4.15) \sim r^{-2.7}$.

(7) The exo-ring scattering structure has a one-sided "leadingedge" arc (or boomerang-like) morphology suggestive of a bow-shock interaction with the local ISM, as has been seen in several other debris disks.

(8) A "kink" (or "bend") in the southwest side of the leadingedge arc, seen at the location of the SB truncation, may (speculatively) find causality from the close-proximity M-star companion HR 4796B.

Contemporaneous wide-field and narrow-angle diffractionlimited high-contrast coronagraphy in visible light with the highest sensitivity enabled (only) with STIS multi-roll PSF template subtraction provides a uniqueness space to study the small-particle spatial distribution in exoplanetary debris systems over a very wide stellocentric distance range. This capability is unduplicated with ground-based high-contrast imaging systems. To date, however, this capability has been exploited at its highest levels of sensitivity and fidelity with only a very small number of exoplanetary debris systems studied, as exemplified here with HR 4796A. With many, if not most, technical challenges now understood and addressed, this capability should be used to its fullest prior to the end of the HST mission to establish a legacy of the most robust images of high-priority exoplanetary debris systems as an enabling foundation for future investigations in exoplanetary systems science.

Based on observations made with the NASA/ESA Hubble Space Telescope, obtained at the Space Telescope Science
Institute (STScI), which is operated by the Association of Universities for Research in Astronomy, Inc., under NASA contract NAS 5-26555. These observations are associated with program \#13786. Support for program \#13786 was provided by NASA through a grant from STScI.

\section{ORCID iDs}

Glenn Schneider (10 https://orcid.org/0000-0002-4511-5966

John H. Debes (iD https://orcid.org/0000-0002-1783-8817

Andras Gáspár (iD https://orcid.org/0000-0001-8612-3236

Dean C. Hines (iD https://orcid.org/0000-0003-4653-6161

Marc J. Kuchner (iD https://orcid.org/0000-0002-9622-9605

Marshall Perrin (iD https://orcid.org/0000-0002-3191-8151

John P. Wisniewski (i) https://orcid.org/0000-0001-9209-1808

\section{References}

Apai, D., Schneider, G., Grady, C. A., et al. 2015, ApJ, 800, 136 Augereau, J. C., Lagrange, A. M., \& Mouillet, D. 1999, A\&A, 350, L51 Boccaletti, A., Thalmann, C., Lagrange, A-M, et al. 2015, Natur, 526, 230 Bonnefoy, M., Milli, J., Ménard, F., et al. 2017, A\&A, 597, L7 Chiang, E., Kite, E., Kalas, P., et al. 2009, ApJ, 693, 734 Clampin, M., Krist, J. E., Ardilla, D. R., et al. 2003, ApJ, 126, 385 Debes, J., Potee, C. A., Jang-Condell, H., et al. 2017, ApJ, 835, 205 Debes, J., Weinberger, A. J., Kuchner, M. J., et al. 2009, ApJ, 702, 318 Debes, J., Weinberger, A. J., Schneider, G., et al. 2008, ApJ, 673L, 191 ESO 2014, http://eso.org/public/usa/news/eso1417/

Feldt, M., Olofsson, J., Boccaletti, A., et al. 2017, A\&A, 601, 7 Gáspár, A., Su, K. Y. L., Rieke, G. H., et al. 2008, ApJ, 672, 974 Gerbaldi, M., Faraggiana, R., Burnage, R., et al. 1999, A\&AS, 137, 273 Grady, C. A., Proffitt, C. R., Malimuth, E., et al. 2003, PASP, 115, 1036 Heap, S. R., Lindler, D. J., Lanz, T. M., et al. 2000, ApJ, 539, 435 Henyey, L. G., \& Greenstein, J. L. 1941, ApJ, 93, 70

Hines, D. C., Schneider, G., Hollenbach, D., et al. 2007, ApJ, 671, 165 Hog, E., Fabricius, C., Makarov, V. V., et al. 2000, A\&A, 355, 27 Houk, N. 1982, Michigan Spectral Survey (Ann Arbor, MI: Univ. Michigan Press), 3

Huélamo, N., Fernández, M., Neuhäuser, R., et al. 2004, A\&A, 428, 953 Huélamo, N., Neuhäuser, R., Seltzer, B., et al. 2000, A\&A, 359, 227 Jura, M. 1995, ApJ, 445, 451

Konishi, M., Grady, C. A., \& Schneider, G. 2016, ApJ, 818L, 32

Krist, J. E., Hook, R. N., \& Stoehr, F. 2011, Proc. SPIE, 8127, 81270J Lagrange, A-M, Milli, J., Boccaletti, A., et al. 2012, A\&A, 546, 38 Maness, H. L., Kalas, P., Peek, K. M. G., et al. 2009, ApJ, 707, 1098 Milli, J., Mouillet, D., Lagrange, A-M, et al. 2012, A\&A, 545, 111 Moór, A., Curé, M., Kóspál, Á., et al. 2017, ApJ, 849, 123

Pascual, N., Montesinos, B., Meeus, G., et al. 2016, A\&A, 586, A6 Perrin, M. D., Duchene, G., Millard-Blancher, M., et al. 2015, ApJ, 799, 182 Perrot, C., Boccaletti, A., Pantin, E., et al. 2016, A\&A, 590, 7 Redfield, S., \& Linsky, J. L. 2008, ApJ, 637, 283

Riley, A. 2017, STIS Instrument Handbook, Version 16.0 (Baltimore, MD: STScI) Rodigas, T. J., Malhorta, R., Hinz, P. M., et al. 2014, ApJ, 780, 65 Rodigas, T. J., Stark, C. C., Weinberger, A., et al. 2015, ApJ, 798, 96 Schneider, G. 2001, AAS, 198, 8301

Schneider, G., Gáspár, A., Debes, J., et al. 2017, Instrument Science Report STIS 2017-3, http://adsabs.harvard.edu/abs/2017stis.rept....3S Schneider, G., Grady, C. A., Hines, D. C., et al. 2014, AJ, 148, 59 Schneider, G., Grady, C. A., Stark, C. C., et al. 2016, AJ, 152, 64 Schneider, G., Smith, B. A., Becklin, E. E., et al. 1999, ApJ, 513L, 127 Schneider, G., Weinberger, A. J., Becklin, E. E., et al. 2009, AJ, 137, 53 Stark, C., Schneider, G., Weinberger, A. J., et al. 2014, ApJ, 789, 58 Stauffer, J. R., Hartmann, L. W., \& Barrado y Navascues, D. 1995, ApJ, 454, 910 Thalmann, C., Janson, M., Buenzli, E., et al. 2011, ApJ, 743, 6 Thebault, P., Marzari, F., \& Augereau, J.-C. 2010, A\&A, 524, 13 Thilliez, E., \& Maddison, S. T. 2016, MNRAS, 457, 1690 Van Leeuwen, F. 2007, A\&A, 474, 653

Wahhaj, Z., Liu, M. C., Biller, B. A., et al. 2014, A\&A, 567, 34 Weinberger, A. J., Becklin, E. E., Schneider, G., et al. 1999, ApJL, 525, L53 Weinberger, A. J., Rich, R. M., Becklin, E. E., et al. 2000, ApJ, 544, 937 